\title{
Auctions and Finite Posted Prices in Online Markets
}

\author{
by \\ Alexander Maslov \\ A thesis submitted to the Faculty of Graduate and Postdoctoral Affairs in partial \\ fulfillment of the requirements for the degree of \\ Doctor of Philosophy \\ in \\ Economics \\ Carleton University \\ Ottawa, Ontario
}

(C) 2017

Alexander Maslov 


\begin{abstract}
The thesis presents a model of a competing mechanism allowing buyers already engaged in an auction to decide whether to stay in it or buy an object from a posted price outside.
\end{abstract}

In the first chapter I describe the relevance of the studied problem to the real world online platforms and discuss how my research contributes to the existing literature. I analyze buyers' game and show that when the amount of goods outside is less than the number of buyers, the latter, with valuations higher than the posted price, prefer to leave the auction early and buy the good at the posted price.

In the second chapter I analyze seller's revenues and show that contrary to the models with effectively infinite goods offered at posted prices and auctions simultaneously, using mixed mechanism may not always be a profit maximizing strategy on markets with one seller and limited supply of goods. When there is competition among sellers, mixed mechanism is the equilibrium. However, sellers do not use it to discriminate between low- and high-valuation buyers as in the models for unlimited supply of goods, but rather as the best response to hedge against the price undercutting by another seller.

In the third chapter I present several extensions to the buyers' subgame equilibrium. I show that the timing of the decision to exit the auction early depends on the degree of risk aversion, number of bidders and the price outside. In addition, I show that the seller's revenues rise with buyers' risk aversion. Lastly, an increase in the number of buyers prompts the seller to use different selling mechanisms. 


\section{Acknowledgements}

I would like to thank David Amundsen, Zhiqi Chen, Eric Stephens, Sergei Severinov and Roland Pongou for their valuable comments and suggestions. I would especially like to thank my supervisors Radovan Vadovič and Aggey Semenov for their support and guidance throughout the work on this thesis. In addition, I would like to point out the indispensable contribution of René Kirkegaard: his comments have helped to transform the thesis substantially. Finally, I am grateful to all other professors and $\mathrm{PhD}$ students with whom I have discussed the ideas developed in this manuscript at conferences, numerous seminars and presentations, which include, but are not limited to Artyom Shneyerov, John List, Elise Critoph, Rami Abou-Seido, Sergiy Pysarenko, Nabil Afodjo, Abdallah Zalghout, Anderson Nzabandora and Matthew Strathearn.

On the administrative side, I am especially grateful to the graduate administrator of my department Marge Brooks — she has helped me immensely throughout the program. I am also grateful to the other administrative staff of the department, who supported me during my tenure: Renee Lortie, Amanda Wright, Sabrina Robineau and Alison Daley. I am thankful to Lynda Khalaf, Patrick Coe and Hashmat Khan who were the supervisors of the Ph.D. program for their guidance. Lastly, my deep thanks go to the departmental administrator Dawn Bjornson and the head of the Department of Economics Chris Worswick for providing me with an opportunity to teach challenging courses and hone my skills as an instructor. 


\section{Contents}

1 Buyers' Game 1

1.1 Introduction . . . . . . . . . . . . . . . . . . 1

1.2 Literature review . . . . . . . . . . . . . . . . . . . 6

1.3 The model . . . . . . . . . . . . . . . . . . . . . . 12

1.3.1 Model set up . . . . . . . . . . . . . . . . . 12

1.3.2 Two buyers . . . . . . . . . . . . . . . 15

1.4 Three buyers . . . . . . . . . . . . . . . . . . . . 17

1.4.1 General case . . . . . . . . . . . . . . . . 17

1.4.2 Uniform distribution . . . . . . . . . . . . . . . . . . 25

2 Sellers' Game $\quad 29$

2.1 One seller (monopoly) . . . . . . . . . . . . . . . 29

2.1.1 Auction - posted price . . . . . . . . . . . . . . . 29

2.1.2 Posted price - posted price . . . . . . . . . . . 33

2.1.3 Auction - auction .................. 38

2.1.4 Discussion . . . . . . . . . . . . . . . . . . . . . . 39

2.2 Two sellers (duopoly) . . . . . . . . . . . . . . . . . 41 
2.2.1 Auction - posted price . . . . . . . . . . . . . . 42

2.2.2 Auction-auction ................... 45

2.2.3 Posted price - posted price . . . . . . . . . . . . . 45

2.2.4 Best responses . . . . . . . . . . . . . . . . 46

2.2.4.1 First seller chooses auction . . . . . . . . . . . 46

2.2.4.2 First seller chooses price . . . . . . . . . . . . 47

2.2.5 Discussion ................... . . . 50

3 Extensions $\quad 53$

3.1 Two objects and $n$ buyers . . . . . . . . . . . . . 53

3.1.1 General case . . . . . . . . . . . . . . . 53

3.1.2 Uniform distribution . . . . . . . . . . . . . . . . 56

3.1.3 Seller's profits . . . . . . . . . . . . . . 60

3.2 Risk aversion . . . . . . . . . . . . . . . . 61

3.2.1 Buyers' equilibrium . . . . . . . . . . . . . . . . . 61

3.2.2 Seller's profits ................... 63

3.3 Not observing the number of players . . . . . . . . . . . . . 65

3.4 Conclusion . . . . . . . . . . . . . . . . . 69

$\begin{array}{ll}\text { Bibliography } & 72\end{array}$

$\begin{array}{ll}\text { Appendices } & 76\end{array}$

$\begin{array}{lll}\text { A Expected payoff of the bidder with valuation } v & 77\end{array}$ 
B Proof of Lemma 3

C Expected seller's revenue from selling both objects as a monopolist 87

$\begin{array}{ll}\text { D Proof of Lemma } 8 & 91\end{array}$

$\begin{array}{ll}\text { E Proof of Lemma } 9 & 96\end{array}$ 


\section{List of Tables}

2.1 Comparing seller's revenues . . . . . . . . . . . . . . . 40

2.2 Sellers' best responses . . . . . . . . . . . . . . . . . . 51 


\section{List of Figures}

1.1 Equilibrium bidding functions. . . . . . . . . . . . . . 19

$1.2 \quad$ Expected payoff. $\ldots \ldots \ldots \ldots \ldots$

1.3 Equilibrium bidding functions and the indifferent buyer. . . . . . . 23

1.4 Equilibrium bidding functions and the threshold price. . . . . . . . 24

2.1 Seller's profit from simultaneously using posted price and auction sell-

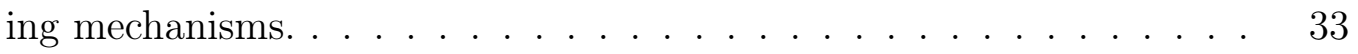

2.2 Two streams of profit from simultaneously using posted prices - low

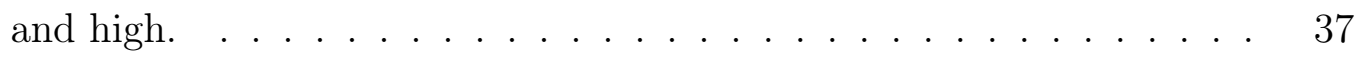

2.3 Decomposed sellers' profits from simultaneously using an auction and

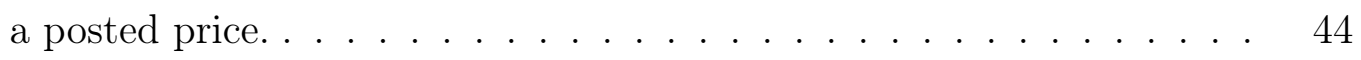

2.4 The continuum of payoffs for sellers using mixed mechanism or posted

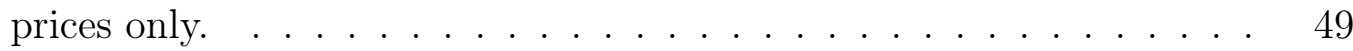

3.1 Simulation of the degree of agreement between original equation and the approximate solution. $\ldots \ldots \ldots \ldots \ldots$

3.2 Simulation of profits from different mechanisms for different number of

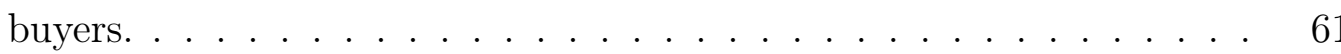


3.3 Simulation of profits and optimal prices for different coefficients of reverse degree of relative risk aversion. . . . . . . . . . . 64 


\section{Chapter 1}

\section{Buyers' Game}

\section{$1.1 \quad$ Introduction}

The last decades have been characterized by rampant growth of the Internet and its associated technologies. One of the most salient examples of such development is e-commerce, which has been showing double-digit growth numbers and is still far from depleting its potential. Internet sales have not only changed the structure of certain retail markets (for example, the recent closure of Sears in Canada), but also gave rise to new technological companies, which would position themselves as buffers and catalysts of online trade (e.g. Shopify, Bigcommerce or Volusion). Of course, most attention was received by large online trading platforms like eBay, Amazon, Alibaba, Etsy, Rakuten or Bonanza. One of the key characteristics of online platforms is the possibility to sell goods by either posted prices or auctions. While some platforms decided to retire auctions (Amazon) and others - on the contrary, emphasize them (eBid), both selling mechanisms are still present in global and local online markets. 
E-commerce may roughly be separated into three segments: business-to-business (B2B), business-to-consumer (B2C) and consumer-to-consumer (C2C). Some online platforms concentrate only on one segment of e-commerce (e.g. DHgate or eWorldTrade for B2B) while others prefer to encompass several or all the segments (e.g. eBay or Alibaba). In many instances, there is very high differentiability of the goods sold on those markets. Nevertheless, consumers still see some products as unique and are not easily diverted into similar products. Hence, while the competitive constraints exist in online markets, in many segments consumers have to compete for effectively limited number of unique objects. In many cases the number of buyers desiring a particular good is higher than the number of available goods.

In the manuscript I analyze how the presence of finite posted prices affects the behavior of buyers in an auction and sellers' profits in two-sided online markets. By finite posted prices I mean a situation when the number of goods on the market is limited and is less than the number of buyers. I consider the case with two objects ${ }^{1}$ (one offered by a posted price and the other one - by an auction) and three buyers ${ }^{2}$. The crucial feature of this environment is that there are more buyers on the market than the goods offered by auctions and posted prices simultaneously. If the number of goods was infinite or buyers could not switch between the mechanisms during the auction, which was assumed in much of the previous literature, then bidders' strategies would boil down to a choice between committing to an auction and buying the good at the posted price. Under such assumptions a bidder's decision is affected by his

\footnotetext{
${ }^{1}$ Throughout the manuscript the words "object" and "good" are used interchangeably and refer to an effectively homogeneous product on the market.

${ }^{2}$ In the extension I analyze the case of $n$ buyers.
} 
time of arrival to the market ${ }^{3}$ and valuation for the object, which was usually assumed of two types - high and low (Etzion et al. (2006); Caldentey and Vulcano (2007); Sun (2008); Hummel (2015)). I extend this framework to a continuum of types and show how bidders' strategies analyzed in the literature on auctions with a buy-it-now $(\mathrm{BIN})^{4}$ price may be applied in this market context. More precisely, in auctions with a BIN price specified by a seller bidders with high valuations have an incentive to end the auction early by buying the good at the BIN price during the auction ${ }^{5}$. I apply similar incentive structure to the "finite" market and show how it differs from a classic environment of auctions with a BIN price (Mathews and Katzman (2006); Reynolds and Wooders (2009); Chen et al. (2013); Anwar and Zheng (2015)) by deriving general conditions for the existence of equilibrium and providing a closed-form solution for the case when buyers' valuations are distributed uniformly. The latter allows me to further investigate the profits of a seller when he is a monopolist and a duopolist. Each market structure gives rise to different selling mechanisms.

Consider a somewhat stylized model of an Internet sales mechanism in which there is an online market with two goods and three buyers competing for them. If one good is offered by an auction and the other one — by a posted price ${ }^{6}$, then a bidder with high valuation would prefer not to bid his valuation right away, but, based on the number of bidders in the auction and the level of the posted price outside, select some level of the auction price (lower than the posted price outside) at which he would exit the auction and buy the good at the posted price. This decision is motivated by

\footnotetext{
${ }^{3}$ The cost of waiting for buyers is inversely proportional to the time till the end of the auction.

${ }^{4}$ Also sometimes called "buy-now price". Sometimes I will refer to it simply as "BIN auction".

${ }^{5}$ Yahoo and eBay auctions have slightly different BIN prices, which I discuss below.

${ }^{6}$ Assuming that the posted price is higher than the reservation price of the auction.
} 
uncertainty associated with the posted price, which may disappear at any time that he remains in the auction ${ }^{7}$. However, if the situation has changed by the time he makes this decision (which can only happen if the number of active bidders changed or the posted price outside disappeared) he may revise his strategy. For example, if another bidder had dropped out by the time the auction price reached the exit price $^{8}$ of the bidder in question, he would prefer to bid up until the auction price matches the posted price since there is no longer any uncertainty about the posted price outside $^{9}$. On the other hand, if the posted price had disappeared before the auction price reached the exit price of the bidder (signaling that one of the other bidders has left the auction and bought the good outside), his best response would be identical to the standard second-price auction. In essence, the above describes the model I develop in this manuscript. If the number of goods offered on the market was effectively infinite these incentives would not apply.

In reality it is not uncommon to place a bid in an Internet auction also observing certain posted prices, which have an increasing perceived probability of disappearing while the auction runs. I endogenize this probability through the number of observed buyers. In theory, it could have also been done exogenously by assigning some survival function to the posted price which would be correlated with the time the auction runs,

\footnotetext{
${ }^{7}$ The uncertainty originates from the fact that bidders don't know each other valuations, only the distribution. Some bidders may prefer to exit the auction and buy the good at the posted price at any time.

${ }^{8}$ I will use the term "exit price" extensively throughout the manuscript. It characterizes such an auction price at which a bidder's strategy prescribes him to exit the auction and buy the good at the posted price. If a bidder is not able to afford the price outside I will use the term "drop out".

${ }^{9}$ I provide a formal proof in the section on 2 objects and 2 buyers. The intuition, however, is straightforward: if there are only 2 buyers and 2 goods in the market, a buyer can always get one good at most at the posted price given that the reservation price of the auction is below it.
} 
but using the observed number of bidders instead of time of their arrival to the auction allows me to simplify the model by not modeling time explicitly and also to account for the prevalent case of last minute bidding ("sniping"). Moreover, a model when bidders are not able to observe each other does not have a closed-form solution ${ }^{10}$.

The model may offer a theoretical framework for interpreting empirical results observed on markets with auctions and posted prices. In particular, Hammond (2010) finds that sellers with larger inventories prefer to use posted prices only, while Einav et al. (2016) show that recently sellers have started shifting their sales toward posted price channel instead of the auction channel due to the convenience of the former mechanism. Both papers do not separate markets with limited and effectively unlimited supply of goods, but my analysis reveals that posted price selling may be the best strategy due to purely rational reasons. In particular, when the number of objects is not significantly less than the number of buyers and the reservation price of the auction is equal to the marginal cost of the seller, selling goods at posted prices is the best response for a seller who is a monopolist in the market. When there is competition between sellers, the predominance of posted prices in the market may be a sign of collusion since the strategy of posted prices only becomes dominating only when there is no competition or the actions of sellers are coordinated. Competition between sellers gives rise to a mixed mechanism in the equilibrium. Finally, when the difference between the number of buyers and goods on the market is small, using simultaneous auctions reduces sellers' profits substantially, and with time and experience sellers may become more inclined to make a transition to using posted

\footnotetext{
${ }^{10}$ This case is considered in one of the extensions.
} 
prices or a mixed mechanism to sell their goods. In addition, during the last years, online markets have experienced a considerable increase in the number of sellers, which has made the environment of the former similar to my model.

I am not aware of any empirical literature on the simultaneous use of auctions and posted prices on "finite" markets. It would be interesting to test the empirical predictions of this manuscript and separate markets with finite and effectively infinite supply of goods as well as to examine if the proportions of used mechanisms remain the same and the historical trajectories on those markets coincide. Moreover, literature on simultaneous use of auctions and posted prices in the markets with effectively infinite supply of goods considers only one seller. It is not clear if the results will change when there are several sellers competing for buyers as in the model presented here.

The rest of the thesis is structured as follows. Chapter 1 describes related literature and the contribution of the thesis. It describes the theoretical framework of the model and provides a benchmark case for two objects and two buyers as well as characterizes equilibrium strategies for the case of two objects and three buyers. Chapter 2 analyzes seller's revenues for the case of a monopoly and duopoly. Chapter 3 presents several extensions to the model and concludes the thesis.

\subsection{Literature review}

Unlike standard single-good auction models a competing mechanism introduces many sellers competing for buyers. The literature emerged with the separate analysis 
of selling mechanisms in the markets, i.e. competition among auctions or among posted prices (McAfee (1993); Peters and Severinov (1997); Peters and Severinov (2006); Burguet and Sákovics (1999); Virág (2010); Eeckhout and Kircher (2010) and others).

A natural extension to the analysis of selling mechanisms was to reunite them, but the models had to be simplified substantially or even solved numerically ${ }^{11}$. First attempts were made by Etzion et al. (2006) and Caldentey and Vulcano (2007) where the authors model a decision of a buyer arriving to a market between buying the good at a posted price and participating in a second-price sealed auction. They establish a threshold-type buyer based on arrival time and show that buyers with higher valuations buy the good at the posted price and with lower valuations participate in the auction. They also prove that using dual channel ${ }^{12}$ increases seller's profits by providing a form of third degree price discrimination between high- and low-valuation buyers. As in the previous literature buyers have to make a choice between the mechanisms and commit to either of them. In fact, both works pose a question on how the finite number of goods on the market may affect the equilibrium. This manuscript provides a framework for such scenario. A similar environment is considered by Sun (2008). He also considers a market with infinite number of goods being sold at auctions and posted prices simultaneously. The author proves the dependence of buyers' choice between an auction and a posted price on the time of arrival and their valuations. He also shows that using both mechanisms

\footnotetext{
${ }^{11}$ Which is not uncommon to the auction literature, see, for example, Marshall et al. (1994); Hafalir and Krishna (2008).

${ }^{12}$ Using auctions and posted prices simultaneously to sell objects.
} 
simultaneously increases seller's profits.

The above papers assume that the seller offers a sealed bid auction, which is not likely the case in reality. They also rely on heuristics of bidders' behavior and numerical simulations to calculate the revenues from using unilateral or dual channels. Etzion and Moore (2013) analyze the case when sellers offer objects at posted prices and open-bid auctions in which bidders are able to observe their bids and even revise them as the auction continues. They implement opportunity costs for bidding in the auction captured by the delayed time of using the item, which are in some spirit similar to the implicit costs of waiting in my model. Their results are similar to the previous papers which implemented sealed-bid auctions - using dual channel increases the seller's profits. Finally, Hummel (2015) considers a model when an infinite number of objects is sold simultaneously at auctions and posted prices and also shows that in equilibrium there exists a threshold-type buyer separating buyers who choose to buy the good at the posted price and buyers who choose to participate in the auction. In his model the seller prefers to use a mixed mechanism, offering some goods by an auction and others - by a posted price. A mixed mechanism allows him to efficiently price discriminate between high- and low-valuation buyers increasing the revenues. He also shows how the presence of posted prices positively affects the bidding frequency near the end of the auction.

To simplify things I do not use time explicitly and also assume that all buyers start in the auction. However, as discussed later, the time of arrival does not matter if buyers arrive before the auction price reaches their exit price. My thesis contributes to the above literature in several distinct ways. Firstly, I relax the 
assumption of commitment for buyers allowing them to exit the auction and buy the good at the posted price during the auction. Secondly, I place a restriction on the number of available goods which engenders a probability that the good outside might disappear while the auction still progresses. It endows different incentive structure on the bidders, which is similar to the one observed in auctions with a BIN price. Furthermore, I derive a closed-form solution for the uniform distribution of types showing exactly under which conditions a threshold type buyer exists and how the valuations of the other buyers map into their decision to exit the auction and buy the good at the posted price. Lastly, the literature has focused only on one seller, while there are usually several sellers offering the same goods on the markets. I consider a case of strategic interaction between two sellers and show how the mixed mechanism emerges from it.

Etzion and Moore (2013) have already noticed that when the dual channel comprises a posted price and an open-bid auction the incentives for buyers become similar to those observed in auctions with a BIN price. However, they conclude that "existing literature on auctions with a buy-now price cannot predict the performance of the suggested dual channel strategy when considering a seller with effectively unlimited supply". In this manuscript I show how the situation reverses when the supply is finite.

For the last decade there was a number of papers which analyzed auctions with a BIN price for eBay and Yahoo auction formats. At the former it is possible to buy the good at the BIN price before the auction starts or until the bid reaches a "secret" reserve price set by the seller. Yahoo format allows to use BIN option 
during the auction. The incentives of the latter are closer to the ones examined in this manuscript.

Anwar and Zheng (2015) analyze the BIN option for the eBay format. For the case of a single seller they show that the presence of such an option increases seller's expected revenue and reduces the allocative inefficiency. For many sellers the authors prove that between two mechanisms of "auction only" and "auction with a BIN price" the latter is always preferred.

Reynolds and Wooders (2009) prove the existence of a threshold strategy (which is similar to the one I derive in this manuscript) for both eBay and Yahoo auctions and show that when bidders are risk averse, introducing BIN option raises sellers revenues. While the authors analyze the problem of one seller with one object, I consider a market where two objects could be offered by either one or two sellers, and the presence of an outside price affects bidding decisions. I also allow bidders to observe their number during the auction. The latter imposes somewhat different incentive structure on the bidders. For example, if the BIN price is a part of an auction (Yahoo format), then by accepting it a bidder ends the auction. There is no way a bidder with higher valuation could end up without a good (under the assumption of symmetry). In my case, if a bidder with the highest valuation leaves the auction and buys the good at the posted price, the auction still continues, and it will be won by the bidder with the second highest valuation, which might end up paying less than the bidder with the highest valuation. Therefore, it creates allocative inefficiency when the good is awarded to the second bidder with a greater payoff than the one received by the first bidder from buying the good at the posted price. Kirkegaard and 
Overgaard (2008) examine a somewhat similar case of inefficiency arising in sequential auctions. I restrict the analysis to risk neutral buyers, but in the extension show that risk aversion affects bidders' behavior in the same manner as in the literature on auctions with a BIN price. Besides Reynolds and Wooders, the role of varying risk aversion in auctions with a BIN price was also studied by, for example, Mathews and Katzman (2006), Bauner (2015), Chen et al. (2013). Among other results, they all find that buyers' risk aversion prompts bidders to buy the good at the BIN price sooner and increases seller's revenues ${ }^{13}$.

My thesis aims at reuniting two discussed strands of the theoretical literature in the competing mechanisms by showing when and how the incentives from auctions with a BIN price apply in markets with finite goods, which are sold simultaneously by auctions and posted prices. I show that when the seller is a monopolist it is the best response to use posted prices only, while a mixed mechanism provides the highest profits in the case of duopoly. A different approach was exercised in a recent paper where the authors showed how a seller's rate of time impatience may be used to present a unified framework for a seller's decision among using posted prices, regular auctions or auctions with a BIN price (Chen et al. (2016)).

\footnotetext{
${ }^{13}$ In equilibrium BIN price is set with a premium. Hence, buyers utilizing this option pay a higher price for their risk aversion.
} 


\subsection{The model}

\subsubsection{Model set up}

Two homogeneous goods are put up for sale ${ }^{14}$. Later, I will consider two cases: when they are owned by a monopolistic seller, and when each of the objects is sold by an independent seller. One of the goods is offered in a second-price auction; the other one - at a posted price. There are $n \geq 2$ risk neutral buyers with valuations $v_{i}$ who have unitary demands (the valuation for the second object is 0 ) and independent private values that are distributed according to a cumulative distribution function $F$ with support $[\underline{v}, \bar{v}]$ where $\underline{v}$ is the lowest possible realization of a buyer's valuation, while $\bar{v}$ - the highest; $F^{\prime}$ is continuous and positive and $\underline{v} \geq 0$. Let the posted price of the good outside of the auction be $p$ and assume that $\underline{v}<p<\bar{v}$. All buyers arrive to the market at the same time and decide whether they want to buy the good at the posted price or start bidding in the ascending open-bid auction. They have a choice to exit it later at anytime and buy the good at the posted price. Assume also that due to exogenous factors buyers make their decisions sequentially based on their valuation, i.e. the first buyer to choose between bidding in the auction or buying the good at the posted price is the buyer with the highest valuation ${ }^{15}$. Hence, if multiple buyers attempt to buy the object on the outside at the start of the auction,

\footnotetext{
${ }^{14}$ There are two ways in which a dual channel may be modeled: as a clock auction following the incentives a bidder has in each moment of time between $q$ and $q+\Delta q$, where $q$ - is the auction price (e.g. Chen et al. (2013)), or as an open-bid auction when a bidder places a proxy bid committing to it with the possibility to revise it in the future if the auction does not end by that time (e.g. Etzion and Moore (2013)). I follow the latter approach as it turns out to be technically more simple.

${ }^{15} \mathrm{~A}$ similar assumption is made for perfect information environment in the relevant IO literature, and I will discuss it in more details later as it turns out to be crucial in several ways.
} 
the object is assigned to the buyer with the highest valuation. Following Reynolds and Wooders (2009) I do not explicitly model the bidding process ${ }^{16}$, which takes the form of a proxy bidding, similar to eBay: bidders submit their bids, and the price of the auction automatically increases in small increments. The winner is the buyer with the highest bid who gets the object and pays the auction price $q$ equal to the second highest bid. If two bidders simultaneously decide to exit the auction to buy the good at the posted price, only one of them gets it with equal probability while the other bidder remains in the auction ${ }^{17}$. The auction closes once there is only one bidder left in it. He gets the good and pays the second highest bid. To simplify the analysis, reservation price is set to zero.

The model presents a stylized version of an Internet sales mechanism when a bidder makes one bid at the beginning of the auction and then, at the time the auction price reaches it, decides whether he would like to exit the auction or continue bidding $^{18}$. In other words, think of a buyer who places a bid smaller than his valuation and no longer monitors the auction (which would also incur $\operatorname{costs}^{19}$ ), but comes back when the auction price reaches his bid (say, by receiving an e-mail from the auctioneer). Now, he has to decide whether he would like to exit or continue in the auction based on the number of bidders left in it. Hence, there are only two times

\footnotetext{
${ }^{16}$ Also see Van Essen and Wooders (2016).

${ }^{17}$ I use this assumption to avoid rare, but possible cases when bidders have the same valuation and they exit the auction simultaneously. Otherwise, one of them would be left without the good and the possibility to continue bidding in the auction.

${ }^{18}$ There is some evidence that the average number of bids submitted by buyers in eBay auctions is around 2 (Bajari and Hortacsu (2003); Ockenfels and Roth (2006)).

${ }^{19}$ Such setup allows to avoid modeling any costs associated with the bidding process. However, these costs could be introduced into the payoff of buyers the same way as risk aversion - the case discussed in one of the extensions.
} 
when buyers make a decision having observed the auction price (if the auction is still running), the posted price (whether it is still available or not), and the number of active bidders in the auction - at the beginning of the auction and at the time the auction prices reaches their first bid.

To formalize the above, let bidder $i$ 's strategy be a collection of two functions $\beta_{i}=\left\{\beta_{1}, \beta_{2}\right\} . \beta_{1}$ maps bidder's valuation and null history into his bid at the beginning of the auction, i.e. $\beta_{1}:[\underline{v}, \bar{v}] \times H_{0} \rightarrow c_{1}$, where $c_{1}$ is the exit price prescribed by the first bid; and $\beta_{2}$ maps bidder's valuation and positive history into his bid at the time the auction price reaches the bid level specified by the first function $\beta_{1}$, i.e. when $q=c_{1}$. Hence $\beta_{2}:[\underline{v}, \bar{v}] \times H_{c_{1}} \rightarrow c_{2}$, where $c_{2}$ is the exit price prescribed by the second bid. History $H_{\left\{0, c_{1}\right\}}$ is a triple $(n, w, p)$ and includes the following components: number of bidders $n$, number of objects in possession $w=\{0,1,2\}$ and the availability of the posted price $p=\{" A ", " N "\}$, where $p=" A "$ if the posted price is available and $p=" N "$ if it is no longer available. At $H_{0}$ it is always true that $w=0, p=A$ and $n=\tilde{n}$, where $\tilde{n}$ is the initial number of buyers in the market, while at $H_{c_{1}}$ there may be changes to any of the histories. If nothing has changed then it is readily seen that $H_{0}=H_{c_{1}}$ and $c_{1}=c_{2}$.

I restrict the attention to symmetric and (weakly) undominated strategies. By symmetric I mean that all buyers follow the same plan of action. This allows me to drop the subscript $i$. While $\beta$ depends on many parameters I will abuse notation and write down only the most relevant of them for the examined cases. Further restrictions allow to trim the strategy space even further as captured by the lemmas below. 
Lemma 1. If a bidder's valuation is below the posted price or the outside object is not available, bidding own valuation on the first object weakly dominates all other bids.

Proof: This follows from the standard argument (Vickrey (1961)).

Lemma 2. Bidding zero on the second object weakly dominates all other bids.

Proof: I have assumed that a buyer has demand only for a single object, the valuation for the second object is zero. It immediately follows that the buyer would not bid on the second object ${ }^{20}$.

Thus, I am interested in a particular subset of strategy space $\beta_{1}$ described by the definition below. As shown above, for all other cases the strategy is straightforward.

Definition 1. Let $z(v)$ be a subset of the strategy space $\beta_{1}$ when bidders with valuations higher than the posted price have 0 goods and place bids while the posted price is still available in the beginning of the auction.

\subsubsection{Two buyers}

Consider the simplest and rather illustrative case when there are only two buyers $^{21}$. Note that in this case $H_{0}=H_{c_{1}}$, otherwise the auction would have ended before $H_{c_{1}}(q)$. Hence, a bidder places only one bid $c_{1}=c$ in the auction.

\footnotetext{
${ }^{20}$ Note, in equilibrium a buyer would never end up with 2 objects.

${ }^{21}$ The argument readily applies to the case of one buyer.
} 
Proposition 1. There is an equilibrium in which:

$$
\beta(v ; p)=\left\{\begin{array}{lll}
v & \text { if } & v \leq p \\
p & \text { if } & v>p
\end{array}\right.
$$

Beliefs are determined by $\beta^{22}$.

Proof. When $v<p$ the buyer is better off losing the auction and getting zero than buying the object on the outside and getting $v-p<0$. This case is strategically equivalent to the case when the outside price is not available. By Lemma 1 it is true that the buyer bids his valuation $c=v$. In the next case when $v \geq p$ I show that $c=p$ maximizes buyer's expected payoff. To begin, notice that a bidder cannot do better by bidding above $p$ than bidding exactly $p$. This is because the other bidder either stops bidding at $q<p$ if his valuation is less than $p$ or buys the object at exactly $q=p$ if his valuation is higher than $p$. Hence, all bids $v \geq c \geq p$ generate the same expected payoff. It remains to examine the case when $c$ is lower than $p$. Suppose $c=\beta(g)$ where $g \leq p$. In this case the expected payoff is:

$$
\int_{\underline{v}}^{g}(v-x) f(x) d x+\int_{g}^{\bar{v}}(v-p) f(x) d x
$$

First order condition shows when the payoff is at the maximum:

$$
\frac{d}{d g} \int_{\underline{v}}^{g}(v-x) f(x) d x+\frac{d}{d g} \int_{g}^{\bar{v}}(v-p) f(x) d x=0
$$

\footnotetext{
${ }^{22}$ If $q>p$ and the object is still available then the play is off equilibrium path. In that case set beliefs to $v_{j} \sim F[q, \bar{v}]$, however, any other assumption would also work to characterize an off-equilibrium path.
} 
This reduces to $(v-g) f(g)=(v-p) f(g)$ from where it is readily seen that the optimal bid is equal to the outside posted price, i.e. $g=p$.

Thus, in equilibrium each bidder bids up to $\min [v, p]$. If both valuations are higher than the posted price, then at $p$ both buyers simultaneously decide to exit the auction and buy the good at the outside price. One of the buyers is randomly permitted to do so, and the auction closes with the other buyer paying the price $q=p$. Hence, both buyers pay the same price $p$. Function $z(v)$ in figure 1.1 mapping valuations from the $v>p$ domain into the auction price $q$ at which buyers decide to exit the auction (exit price) is constant and parallel to the $v$-axis.

\subsection{Three buyers}

\subsubsection{General case}

Adding one more buyer to the game introduces significant changes. We already know that if $v<p$ it is the best response of a bidder to bid his valuation. In proposition 1 I proved that when there are two buyers and two objects in a market, it is the best response for a bidder to bid up to his own valuation or up to the auction price which matches the posted price outside (whichever happens first). When the number of buyers is higher than the number of goods available outside it is easy to show that for bidders with valuations higher than the posted price bidding up to $q=p$ can no longer be the equilibrium. Suppose that all three bidders have valuations higher than the posted price. If they follow the strategy from the $2 \mathrm{x} 2$ case then at 
the price $q=p$ one of the bidders wins the auction and pays $q=p$, another bidder attempts to buy the object at the posted price $p$ and gets it with some probability while the remaining bidder is left without an object and with a payoff of 0 . The latter bidder can improve his payoff by exiting the auction right before the price reaches $p$ and buying the good outside. On the other hand, other bidders can do the same at a price lower than the price at which the first bidder exits. Therefore, there exists some auction price $q=c<p$ at which a bidder decides whether he wants to exit the auction or continue bidding. Following the description of the model, the initial bid submitted by the bidder is equal to this level of the auction price $c=c_{1}$. At this price, he comes back to the auction and either exits it or continues bidding if the history at that point has changed.

It is easy to see that if one player drops out from the auction before $q$ reaches $c$, the equilibrium of the $3 \times 2$ game becomes identical to that of the $2 \times 2$ case - bidders no longer exit the auction, but rather bid their valuations up to the posted price. According to Lemma 1, if one player exits the auction and buys the good at the posted price, the remaining players bid their valuation.

Similar to Reynolds and Wooders (2009), the payoffs of buyers depend on the profile of strategies and the maximum of other buyers' valuations (figure 1.1). To find the function mapping valuations into the exit price I will define the expected payoff of the bidder with valuation $v$ and maximize it with respect to the exit price as I did in the $2 \mathrm{x} 2$ case $^{23}$. With out loss of generality let $v$ denote the valuation of the first

\footnotetext{
${ }^{23}$ See Appendix A for a detailed construction of the objective function.
} 
bidder, $x$ - second bidder and $y$ - third bidder. There are several possibilities ${ }^{24}$ :

Figure 1.1: Equilibrium bidding functions.

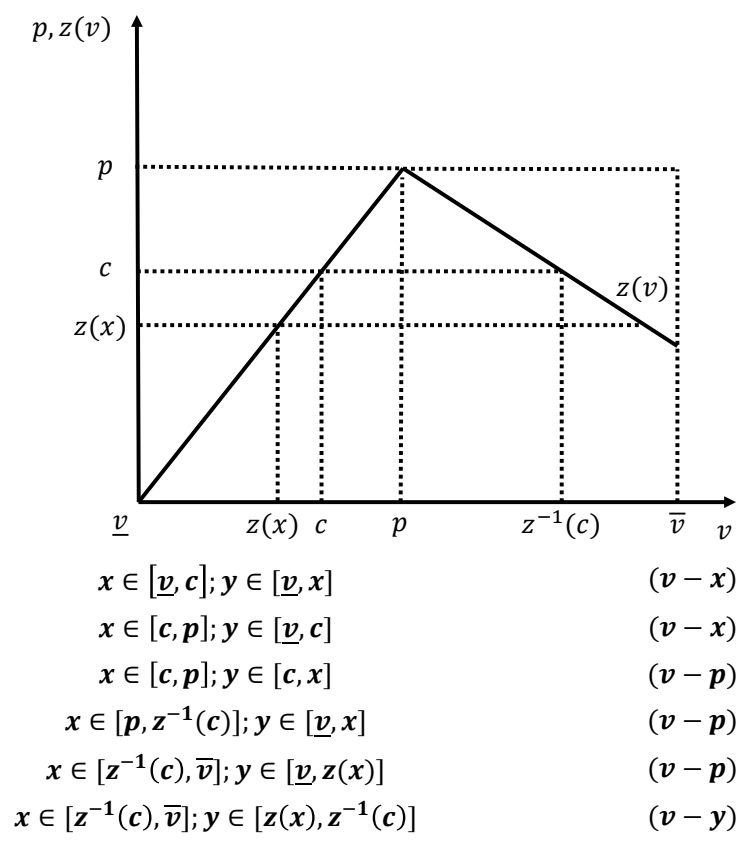

Figure 1.2: Expected payoff.

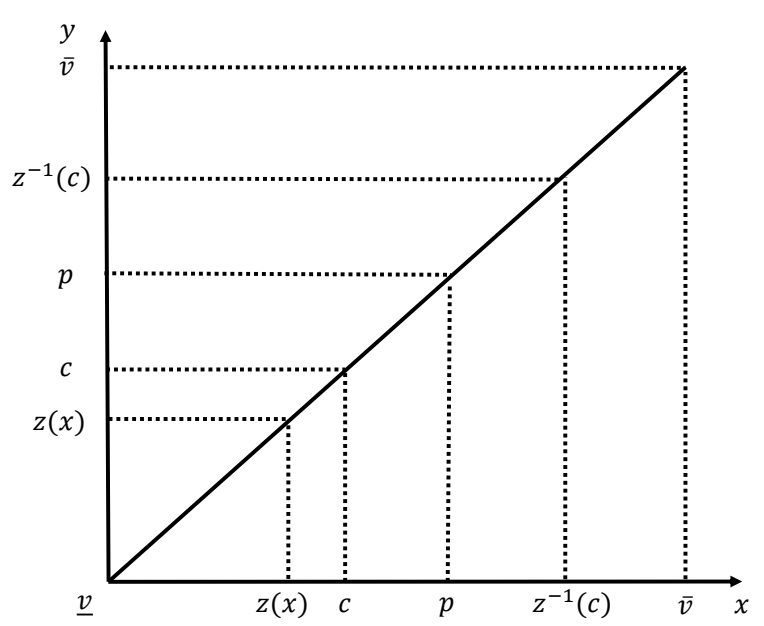

${ }^{24}$ Figure 1.2 presents another exposition of the payoff structure. 


$$
\begin{gathered}
\max _{c}\left(\int_{\underline{v}}^{c} \int_{\underline{v}}^{x}(v-x) 2 f(x) f(y) d y d x+\int_{c}^{p} \int_{\underline{v}}^{c}(v-x) 2 f(x) f(y) d y d x+\right. \\
+\int_{c}^{p} \int_{c}^{x}(v-p) 2 f(x) f(y) d y d x+\int_{p}^{z^{-1}(c)} \int_{\underline{v}}^{x}(v-p) 2 f(x) f(y) d y d x+ \\
\left.+\int_{z^{-1}(c)}^{\bar{v}} \int_{\underline{v}}^{z(x)}(v-p) 2 f(x) f(y) d y d x+\int_{z^{-1}(c)}^{\bar{v}} \int_{z(x)}^{z^{-1}(c)}(v-y) 2 f(x) f(y) d y d x\right)
\end{gathered}
$$

The differential equation resulting from the maximization problem above does not seem to have a closed-form solution unless the distributional function is specified, but I can rewrite it in the form of a corresponding slope field:

\section{Lemma 3.}

The derivative of the equilibrium exit function mapping bidder's valuation into the exit price for all $v>p$ is described by the following equation ${ }^{25}$ :

$$
z^{\prime}(v)=\frac{f(v)}{f(c)} \frac{p(F(v)-F(c))-\int_{c}^{v} y f(y) d y}{F(c)(c-p)+\int_{c}^{p} F(x) d x}
$$

Having the following properties:

a) The function $z(v)$ is bounded upwardly by $p$.

b) The slope of the function $z(v)$ is negative if and only if $p<E(y) \mid y \in[c, v]$, in other words, a bidder exits the auction before its price matches the posted price outside if and only if the expectation of the lowest bidder's valuation between the value of the exit function and the valuation of the former bidder is higher than the posted price.

\footnotetext{
${ }^{25}$ Note that in equilibrium $z(v)=c$, but I retain $c$ to simplify notation and enhance intuition.
} 
c) The slope of the function $z(v)$ with respect to $p$ is negative.

Proof. Appendix B.

I have derived conditions which ensure the existence of the function mapping bidders' valuations into the exit price. I also showed that the slope changes in the opposite direction to the changes in the posted price.

The condition for the function to be negative is intuitive - it means that in order for the first bidder with valuation $v$ to leave the auction before its price reaches the level of the posted price, the expected value of the third bidder with the lowest valuation $y$ should be greater than the posted price. If this condition was not satisfied then the first bidder would expect the third bidder to drop out from the auction before the auction price matched the posted price outside. If the second bidder's valuation is in $\frac{\int_{c}^{v} y f(y) d y}{F(v)-F(c)}<x<p$ then the first bidder gets a payoff greater than he would have if he left the auction. On the other hand, if the second bidder's valuation is higher than the posted price $(x>p)$, the first bidder does not lose anything by waiting till the auction price matches the posted price and then buying the good outside (or in the auction if the second bidder is the first to exit). In other words, if $p<E(y)$ on $[c, v]$ is not satisfied, the first bidder plays the $2 \mathrm{x} 2$ case and bids according to its best strategy.

Before formulating the final equilibrium for the $3 \times 2$ case I would like to elaborate on the assumption I stated earlier (Chen et al. (2013) have a similar assumption in their paper, which allows them to find a closed-form solution). 
Assumption 1. If several bidders choose to buy the good at the posted price at the beginning of the auction then the good is awarded to the bidder with the highest valuation.

In the model setup I assumed that buyers make a bid or buy the good at the posted price sequentially based on their valuation ${ }^{26}$. The assumption is a natural extension to this reasoning. It is a rule of rationing which simplifies the analysis. It traces back to the IO literature which deals with competition in prices and allows to ration the demand between sellers when their capacities are limited (Levitan and Shubik (1972); Kreps and Scheinkman (1983); Davidson and Deneckere (1984); Osborne and Pitchik (1986); Vives (1986)). My model is in some spirit similar to the capacity constraints models since both sellers have only one good.

The assumption is also required to avoid a well-documented discontinuity in the equilibrium function $z(v)$ (as in, for example, Mathews and Katzman (2006) or Reynolds and Wooders (2009)). If it was not the case then when $z(v)=0$ the probability of getting the good outside for a bidder who does not have the highest valuation would be positive (which is not the case for $z(v)>0$ given the symmetric nature of the equilibrium). It creates an incentive for other bidders with even lower valuations to deviate towards buying the good right at the beginning of the auction, which makes it significantly harder to derive the valuation of the threshold bidder and even more so using it in the sellers' game. In the literature on BIN auctions it is

\footnotetext{
${ }^{26}$ Note that it is not the mechanism that defines which bidder has the highest valuation, but it is predetermined by exogenous forces, for example, a bidder with the highest valuation values the good higher than the others and is always the fastest to get it if several bidders decide to exit at the start of the auction. In addition, he spends more time searching and monitoring for the good he desires, which allows him to be the first one to get it.
} 
usually given implicitly. This assumption is also important because it does not allow to use the mechanism design approach by imposing exogenous efficiency ${ }^{27}$.

The assumption allows to find a range of posted prices for which function $z(v)$ intersects $v$-axis (figure 1.3). Intuitively, it identifies a valuation of a buyer $(\hat{v})$ who is indifferent between bidding in the auction and buying the good outside at the start of the auction. If a buyer's valuation is higher than the valuation of the indifferent buyer, he will opt-out for the posted price right at the beginning of the auction. I will find this range of prices for the uniform distribution case examined below. When function $z(v)$ does not intersect the $v$-axis and $v>p$ the equilibrium is completely determined by $z(v)$. Let $\tilde{p}$ be such a price that for all $p>\tilde{p}$ indifferent bidder does not exist (figure 1.4). Then the equilibrium for the $3 \times 2$ case could be stated by the following proposition:

Figure 1.3: Equilibrium bidding functions and the indifferent buyer.
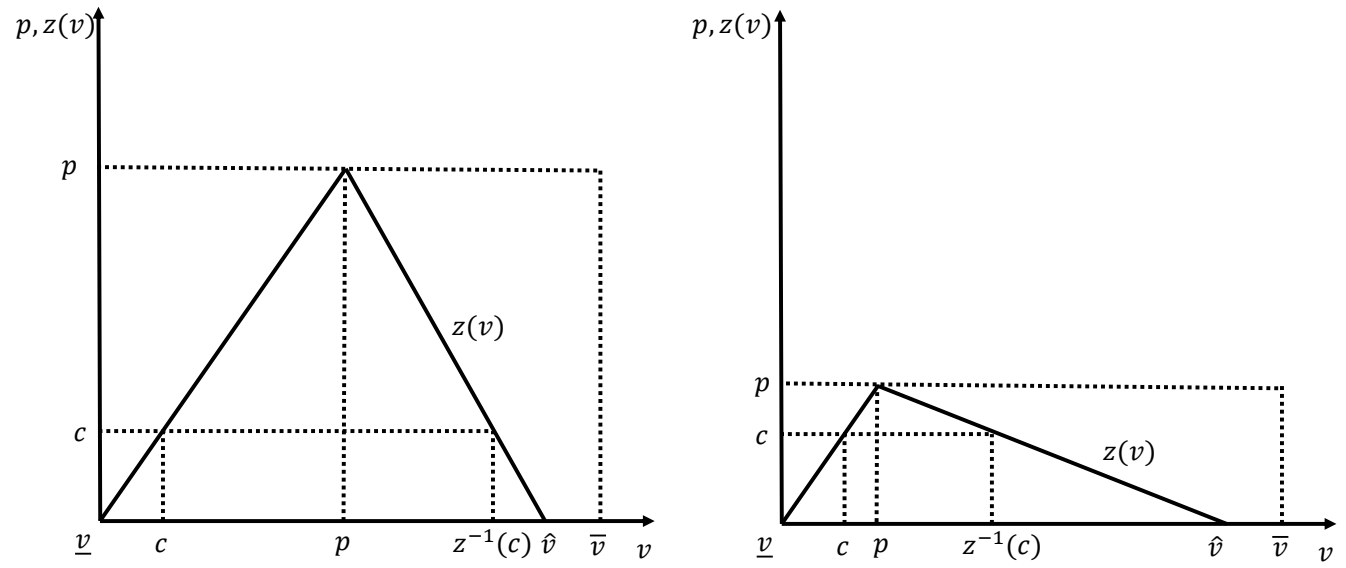

\footnotetext{
${ }^{27}$ I would like to especially thank René Kirkegaard for pointing this out. I will provide an example of it when analyzing seller's game.
} 
Figure 1.4: Equilibrium bidding functions and the threshold price.

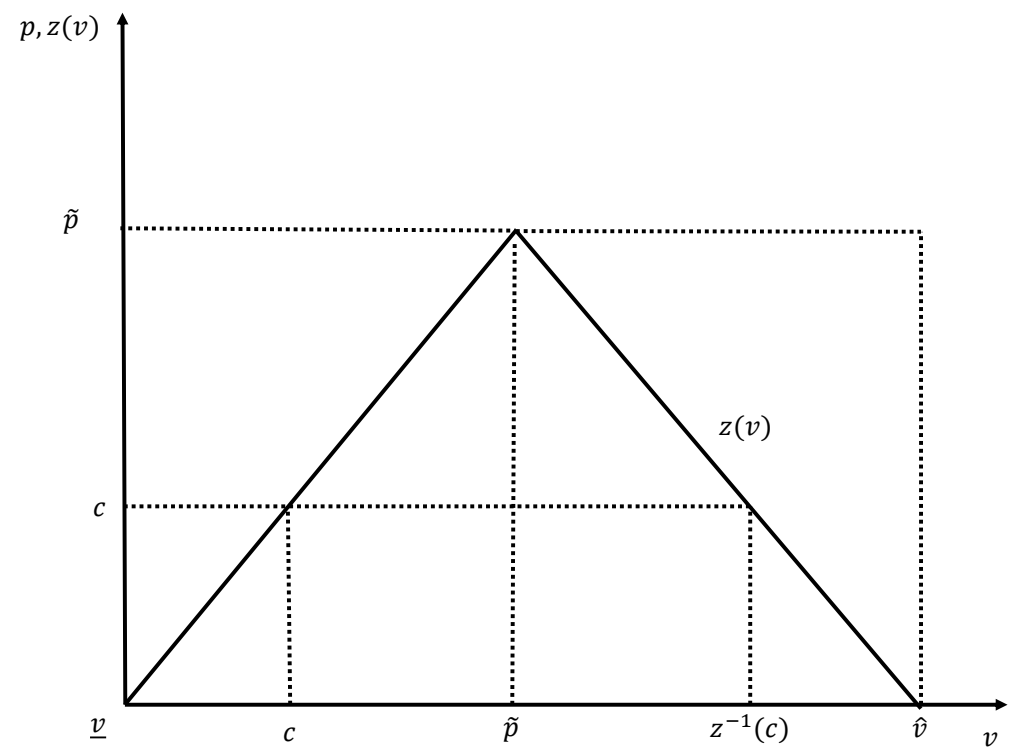

Proposition 2. There is an equilibrium in which:

$$
\beta(v, p)= \begin{cases}v & \text { if } v \leq p \\ z(v) \text { with } z^{\prime}(v) \text { defined by (1.4) } & \text { if } v>p \text { and } p \geq \tilde{p} \\ z(v) \text { with } z^{\prime}(v) \text { defined by (1.4) } & \text { if } p<v<\hat{v} \text { and } p<\tilde{p} \\ 0 & \text { if } \hat{v} \leq v \leq \bar{v} \text { and } p<\tilde{p}\end{cases}
$$

Where $\hat{v}$ is the valuation of the indifferent bidder and $\tilde{p}$ is the threshold price for the existence of the indifferent bidder. Beliefs are completely determined by $\beta^{28}$.

\footnotetext{
${ }^{28}$ If the posted price disappeared and there are still 3 bidders in the auction then the play is off equilibrium path. In that case set beliefs to $\sum_{j=1}^{2} v_{j} \sim F[p, \bar{v}]$, however, any other assumption would also work to characterize an off-equilibrium path.
} 


\subsubsection{Uniform distribution}

Assuming that the distribution function from which valuations are drawn is uniform on $[0,1] \mathrm{I}$ can plug in corresponding PDFs $(f(\psi)=1)$ and $\operatorname{CDFs}(F(\psi)=\psi)$ into (1.4) and after rearranging receive:

$$
(p-z(v))^{2}+\frac{(v-z(v))(v+z(v)-2 p)}{z^{\prime}(v)}=0
$$

Lemma 4. For all $v>p$ and $p>\frac{3}{7}$ the exit function is a monotonic and decreasing function of the exit price level: $\frac{7}{4} p-\frac{3}{4} v$. For $v>p$ and $p \leq \frac{3}{7}$ the equilibrium exit function has a kink at $z(v)=0$.

Proof. Rewrite equation (1.6) as follows (to simplify notation I will write $z$ instead of $z(v)$, also note that $\left.(p-z)^{2}=(z-p)^{2}\right)$ :

$$
(z-p)^{2} z^{\prime}+(v-z)(v+z-2 p)=0
$$

Substituting $Z=z-p$ and $V=v-p$ gives:

$$
\begin{gathered}
Z^{2} Z^{\prime}+(V-Z)(V+Z)=0 \\
\frac{d Z}{d V}=Z^{\prime}=\frac{Z^{2}-V^{2}}{Z^{2}}
\end{gathered}
$$


Replace $Z=V F(V)$ to get:

$$
\begin{gathered}
V \frac{d F}{d V}+F=\frac{F^{2}-1}{F^{2}} \rightarrow V \frac{d F}{d V}=\frac{F^{2}-1-F^{3}}{F^{2}} \\
\frac{d V}{V}=\frac{F^{2}}{-F^{3}+F^{2}-1} d F \rightarrow \ln (V)=\int \frac{F^{2}}{-F^{3}+F^{2}-1} d F
\end{gathered}
$$

The denominator $-F^{3}+F^{2}-1$ can be decomposed using three roots - two complex and one real:

$$
\begin{gathered}
\int \frac{F^{2}}{-\left(F-r_{1}\right)\left(F-r_{2}\right)\left(F-r_{3}\right)} d F=-\frac{r_{1}^{2} \ln \left|F-r_{1}\right|}{\left(r_{1}-r_{2}\right)\left(r_{1}-r_{3}\right)} \\
-\frac{r_{2}^{2} \ln \left|F-r_{2}\right|}{\left(r_{2}-r_{1}\right)\left(r_{2}-r_{3}\right)}-\frac{r_{3}^{2} \ln \left|F-r_{3}\right|}{\left(r_{3}-r_{1}\right)\left(r_{3}-r_{2}\right)}+c \\
V=C e^{\left(-\frac{r_{1}^{2} \ln \left|F-r_{1}\right|}{\left(r_{1}-r_{2}\right)\left(r_{1}-r_{3}\right)}-\frac{r_{2}^{2} \ln \left|F-r_{2}\right|}{\left(r_{2}-r_{1}\right)\left(r_{2}-r_{3}\right)}-\frac{r_{3}^{2} \ln \left|F-r_{3}\right|}{\left(r_{3}-r_{1}\right)\left(r_{3}-r_{2}\right)}\right)} \\
V=C\left(\left|F-r_{1}\right|\right)^{\frac{-r_{1}^{2}}{\left(r_{1}-r_{2}\right)\left(r_{1}-r_{3}\right)}}\left(\left|F-r_{2}\right|\right)^{\frac{-r_{2}^{2}}{\left(r_{2}-r_{1}\right)\left(r_{2}-r_{3}\right)}}\left(\left|F-r_{3}\right|\right)^{\frac{-r_{3}^{2}}{\left(r_{3}-r_{1}\right)\left(r_{3}-r_{2}\right)}}
\end{gathered}
$$

In the solution above $F \neq r_{1}, F \neq r_{2}$ and $F \neq r_{3}$ since I assumed these values are not equal to zero when dividing by the corresponding polynomial. From the parametric expression it is seen that when $F$ approaches either of the roots the value of the function becomes infinitely large, which may not be a solution to the bidding function. Hence, I will examine whether the roots of the polynomial are the solutions to the equation (1.8). In other words, I will check if $Z=k V$ solves this equation.

$$
k=\frac{(k V)^{2}-V^{2}}{(k V)^{2}} \Rightarrow k^{3}=k^{2}-1
$$

The last expression is exactly the equation which roots I found. Being only 
interested in the real root the solution is thus $k=-0.7549$ or $Z=-0.7549 \mathrm{~V}$.

Substituting back $Z=z-p$ and $V=v-p$ produces $z-p=-\frac{3}{4}(v-p)$. Solving for $z(v)$ I receive the final equation for the exit function:

$$
z(v)=\frac{7}{4} p-\frac{3}{4} v
$$

The solution is defined only for the case when $v>p$ and it satisfies the condition outlined in the general case, i.e. $p<E(y) \mid y \in[c, v] \Rightarrow p<\frac{z(v)+v}{2} 29$. Note that the function characterizing exit strategy for two sellers and three bidders is linear and negative. Therefore, there is a certain level of price $p$ when it will cross the $v$-axis in the point of an indifferent bidder. To find the threshold $p$ substitute the point $(1,0)$ into the equation (1.6) to get $p=\frac{3}{7}$. Note that the price is relative to the distribution of bidders' valuations. It shows that an indifferent bidder will exist only when $p \leq \frac{3}{7}$. Every bidder with a valuation higher than the indifferent bidder's valuation will not participate in the auction and opt-out for the good at the posted price right at the beginning. According to the assumption 1 a bidder with the highest valuation gets the good at the posted price, and the rest participate in the auction.

\footnotetext{
${ }^{29}$ Substituting $z(v)=\frac{7}{4} p-\frac{3}{4} v$ get $8 p<7 p-3 v+4 v$ or $v>p$.
} 
Proposition 3. There is an equilibrium in which:

$$
\beta(v, p)= \begin{cases}v & \text { if } v \leq p \\ \frac{7}{4} p-\frac{3}{4} v & \text { if } v>p \text { and } p>\frac{3}{7} \\ \frac{7}{4} p-\frac{3}{4} v & \text { if } p<v<\frac{7}{3} p \text { and } p \leq \frac{3}{7} \\ 0 & \text { if } \frac{7}{3} p \leq v \leq 1 \text { and } p \leq \frac{3}{7}\end{cases}
$$

Beliefs are completely determined by $\beta^{30}$.

Proof. To find the value of the indifferent bidder substitute $z(v)=0$ into the equation $(1.12)$, which produces $\hat{v}=\frac{7}{3} p$. The threshold price is defined by Lemma 4 . According to Lemma 1, if a bidder's valuation is less than the posted price outside the auction then the best response is to bid his own valuation. However, Lemma 4 tells us that when his valuation is higher than the posted price, the best response is to leave the auction early and buy the good at the posted price. Moreover, under certain $v$ and $p$ a bidder may not bid in the auction at all.

The form of the function shows that a higher valuation and lower posted price positively affect a buyer's decision to leave the auction early and buy the good at the posted price. Note also that the slope of the exit function for the uniform distribution of valuations does not depend on $p$ even though in the general form it does. It seems that uniform distribution is a particular case when the exit function is linear and it affects the immutability of the slope. Chen et al. (2013) have found a similar closed form solution for the BIN auction with uniform distribution of buyers' valuations.

\footnotetext{
${ }^{30}$ Off-equilibrium path could be specified the same way as in Proposition 2.
} 


\section{Chapter 2}

\section{Sellers' Game}

In this chapter I analyze the revenues of a seller when he is a monopolist or a duopolist on the markets of finite goods.

\subsection{One seller (monopoly)}

\subsubsection{Auction - posted price}

In the previous chapter I found the equilibrium of the buyers' subgame. With the assumption 1 I was able to characterize it in a closed-form solution when buyers' valuations are distributed uniformly. It allows me to further investigate the profit which a seller receives from selling two goods simultaneously using an auction and a posted price ${ }^{1}$. In what follows I assume that the seller is a monopolist, who possesses two goods and wants to sell them to three buyers simultaneously. He has three options: sell both goods by auctions; sell both goods by posted prices; sell one good

\footnotetext{
${ }^{1}$ I have assumed that sellers' marginal costs are zero, so profits are equivalent to revenues.
} 
by an auction and the other one - by a posted price.

To formulate the expected profit of the seller I need to again consider all regions from the distribution of buyers' valuations. As shown by the proposition 3 , conditional on the level of the posted price set by the seller, the exit function for bidders may either have a kink where it crosses $v$-axis or not. Previously, I found that when $p>\frac{3}{7}$ a bidder's exit function does not have a kink, but when $p \leq \frac{3}{7}-$ it does. Therefore, I will consider two different regions described by two profit functions: one for $p \in\left[0, \frac{3}{7}\right]$, when an indifference bidder exists; and the other one for $p \in\left(\frac{3}{7}, 1\right]$, when the indifference bidder does not exist.

Lemma 5. If $p \in\left[0, \frac{3}{7}\right]$ then the profit function of the seller is described by the following equation: $\pi_{1}^{A P}=\frac{1}{4}+p-\frac{13}{12} p^{4}$.

Proof. From the equilibrium of the buyers' subgame it is known that there are certain values of $v$ for which a buyer with the highest valuation exits the auction right at the beginning. The range of such values belongs to the region $\left[\frac{7}{4} p-\frac{3}{4} v, 1\right]$. In proposition 3 I showed that the value of the indifference buyer is defined by $\hat{v}=\frac{7}{3} p$. Keeping the same notation for the buyers (without loss of generality $v$ - buyer with the highest valuation, $x$ - buyer with the second highest valuation, $y$ - buyer with the lowest valuation) there are several possibilities of realization of their valuations ${ }^{2}$.

The detailed construction of the expected profit is done in the Appendix C. The expected profit of the seller when $0 \leq p \leq \frac{3}{7}$ could be expressed as follows (note

\footnotetext{
${ }^{2}$ Figure 1.1 is again helpful in understanding different regions of distribution of buyers' valuations. In particular, there are 5 regions which should be investigated in this case: $[0, c],[c, p],[p, v]$ and $[v, \hat{v}]$ and $[\hat{v}, 1]$.
} 
that $\left.c=\frac{7}{4} p-\frac{3}{4} v\right)$ :

$$
\begin{gathered}
\pi_{1}^{A P}=6\left[\int_{0}^{p} \int_{0}^{v} \int_{0}^{x} x d y d x d v+\int_{p}^{\frac{7}{3} p} \int_{0}^{\frac{7}{4} p-\frac{3}{4} v} \int_{0}^{x} x d y d x d v+\right. \\
\int_{p}^{\frac{7}{3} p} \int_{\frac{7}{4} p-\frac{3}{4} v}^{p} \int_{0}^{\frac{7}{4} p-\frac{3}{4} v} x d y d x d v+\int_{p}^{\frac{7}{3} p} \int_{\frac{7}{4} p-\frac{3}{4} v}^{p} \int_{\frac{7}{4} p-\frac{3}{4} v}^{x}(y+p) d y d x d v+ \\
\int_{p}^{\frac{7}{3} p} \int_{p}^{v} \int_{0}^{\frac{7}{4} p-\frac{3}{4} v} 2 p d y d x d v+\int_{p}^{\frac{7}{3} p} \int_{p}^{v} \int_{\frac{7}{4} p-\frac{3}{4} v}^{p}(y+p) d y d x d v+ \\
6\left[\frac{p^{4}}{12}+\frac{p^{4}}{9}+\frac{p^{4}}{6}+\frac{p^{4}}{3}+\frac{16 p^{4}}{27}+\frac{26 p^{4}}{27}+\frac{224 p^{4}}{243}+\frac{81+324 p-6517 p^{4}}{1944}\right]
\end{gathered}
$$

Simplifying, the expected profit of the seller when $0 \leq p \leq \frac{3}{7}$ is equal to:

$$
\pi_{1}^{A P}=\frac{1}{4}+p-\frac{13}{12} p^{4}
$$

Note that when $p=0$ the expected profit of the seller is equal to $\frac{1}{4} \cdot{ }^{3}$

Lemma 6. If $p \in\left(\frac{3}{7}, 1\right]$ then the profit function of the seller is described by the following equation: $\pi_{2}^{A P}=-\frac{71}{256}+\frac{235 p}{64}-\frac{429 p^{2}}{128}-\frac{77 p^{3}}{64}+\frac{425 p^{4}}{256}$.

Proof. When the posted price is set in the region $p \in\left(\frac{3}{7}, 1\right]$ the threshold bidder does not exist and, by an analogy with the analysis above, the profit of the seller could be formulated as follows:

\footnotetext{
${ }^{3}$ This is a good example to show that the mechanism design approach is not applicable. Since buyers' valuations are distributed uniformly the virtual valuation of a buyer is equal to $J(v)=2 v-1$. If $p=0$ then the good at the posted price goes to the bidder with the highest valuation and the auctioned good is allocated to the buyer with the second highest valuation (according to the assumption 1). Hence, buyer with valuation $x$ wins one unit unless he has the lowest valuation: $3 \int_{0}^{1}(2 x-1)\left(1-(1-x)^{2}\right) d x=\frac{1}{2}$, which is not equal to $6 \int_{0}^{1} \int_{0}^{v} \int_{0}^{x} y d y d x d v=\frac{1}{4}$.
} 


$$
\begin{gathered}
\pi_{2}^{A P}=6\left[\int_{0}^{p} \int_{0}^{v} \int_{0}^{x} x d y d x d v+\int_{p}^{1} \int_{0}^{\frac{7}{4} p-\frac{3}{4} v} \int_{0}^{x} x d y d x d v+\right. \\
\int_{p}^{1} \int_{\frac{7}{4} p-\frac{3}{4} v}^{p} \int_{0}^{\frac{7}{4} p-\frac{3}{4} v} x d y d x d v+\int_{p}^{1} \int_{\frac{7}{4} p-\frac{3}{4} v}^{p} \int_{\frac{7}{4} p-\frac{3}{4} v}^{x}(y+p) d y d x d v+ \\
\int_{p}^{1} \int_{p}^{v} \int_{0}^{\frac{7}{4} p-\frac{3}{4} v} 2 p d y d x d v+\int_{p}^{1} \int_{p}^{v} \int_{\frac{7}{4} p-\frac{3}{4} v}^{p}(y+p) d y d x d v+ \\
\left.\int_{p}^{1} \int_{p}^{v} \int_{p}^{x}(y+p) d y d x d v\right]=-\frac{71}{256}+\frac{235 p}{64}-\frac{429 p^{2}}{128}-\frac{77 p^{3}}{64}+\frac{425 p^{4}}{256}
\end{gathered}
$$

Note that when $p=1$ the expected profit is equal to $\frac{1}{2}$.

Proposition 4. The seller maximizes his profits from the mixed mechanism by setting $p=0.550$ and earning $\pi^{A P}=0.680$.

Proof. The seller wants to set such a price that would maximize his profit. Check both regions for the existence of such a price. When $\frac{d \pi_{1}^{A P}}{d p}=0$ seller's profit is maximized at $p=0.613$, which is outside the region where the function is defined ${ }^{4}$. When $\frac{d \pi_{2}^{A P}}{d p}=0$ the profit is maximized at $p=0.550$ which satisfies the condition. The profit at this price is equal to 0.680 . The profit functions are presented on figure 2.1 .

\footnotetext{
${ }^{4}$ Notice that $\pi_{1}^{A P}$ is an increasing function on $\left[0, \frac{3}{7}\right]$. Hence, the corner solution would be in the vicinity of $p=\frac{3}{7}$ and approximately equal to 0.643 .
} 
Figure 2.1: Seller's profit from simultaneously using posted price and auction selling mechanisms.

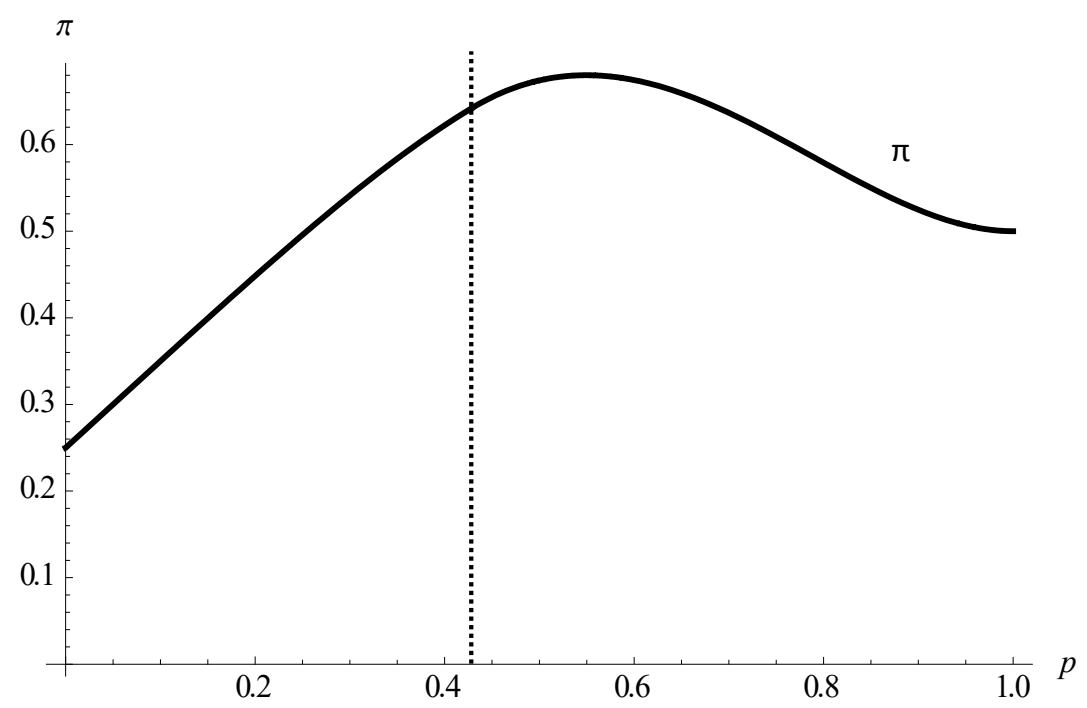

I have established that the maximum profit a seller could get when simultaneously using an auction and a posted price to sell his goods is equal to $0.680^{5}$. Below I will find profits from the other variations of selling mechanisms and compare them to the profit received under the simultaneous use of an auction and a posted price.

\subsubsection{Posted price - posted price}

When both objects are sold by posted prices it is straightforward to calculate the expected profit knowing the support of the distribution of buyers' valuations. First, however, I will prove that in the equilibrium a seller uses the same price for both goods.

\footnotetext{
${ }^{5}$ Given that the reservation price is set to 0 , which may not be optimal.
} 
Proposition 5. When simultaneously selling two homogeneous goods to three buyers with uniformly distributed valuations, a seller sets their prices equal to 0.541 in the equilibrium and earns a profit of 0.692 .

Proof. Suppose it was not the case, and the seller would post one good at a low price $p_{l}$ and the other good - at a high price $p_{h}$ (without loss of generality) with $p_{l}<p_{h}$. The expected payoff depends on the implemented rationing rule. Following assumption 1 buyers with higher valuations are the first to attempt to buy the good at the posted price. The expected payoff can be calculated based on different regions of the distribution of buyers' valuations ${ }^{6}$.

a) The first buyer's valuation is above $p_{l}$, but below $p_{h}$, while the other buyers' valuations are below the first buyer's valuation: $p_{l}<v<p_{h} ; x, y<v$. In this case it does not matter if any other buyer's valuation is below or above $p_{l}$, as long as none of the buyers can afford the good at $p_{h}$ the seller will sell only one good to the buyer with the highest valuation. The seller's expected profit is equal to:

$$
\int_{p_{l}}^{p_{h}} \int_{0}^{v} \int_{0}^{x} p_{l} d y d x d v
$$

b) The first buyer's valuation is above $p_{h}$ while the second and the third buyers' valuations are below $p_{l}$, i.e.: $p_{h}<v<1 ; x, y<p_{l}{ }^{7}$. In this case the buyer with the highest valuation buys the good at $p_{l}$ since $v-p_{l}>p-p_{h}$ and the seller's expected profit is:

\footnotetext{
${ }^{6}$ Clearly, if the highest valuation is below $p_{l}$ then no buyer can afford the good and the seller's expected profit is equal to zero.

${ }^{7}$ Note that $v>x>y$. Hence, when I write $x, y<p_{l}$ it means that $0<x<p_{l}$ and $0<y<x$.
} 


$$
\int_{p_{h}}^{1} \int_{0}^{p_{l}} \int_{0}^{x} p_{l} d y d x d v
$$

c) The first buyer's valuation is above $p_{h}$ while the second buyer's valuation is higher than $p_{l}$, but lower than $p_{h}$; the third buyer's valuation is below $p_{l}$, i.e. $p_{h}<v<1$ and $p_{l}<x<p_{h}$ and $0<y<p_{l}$. According to the assumption 1 the buyer with the highest valuation $v$ buys the good and pays the low price. Hence, the buyer with the second highest valuation is not able to afford the good at the high price; seller's revenue is generated only from the low price:

$$
\int_{p_{h}}^{1} \int_{p_{l}}^{p_{h}} \int_{0}^{p_{l}} p_{l} d y d x d v
$$

d) The first buyer's valuation is above $p_{h}$ while the second buyer's valuation is higher than $p_{l}$, but lower than $p_{h}$; third buyer's valuation is higher than $p_{l}$, but below the valuation of the second buyer. In this scenario the good is again awarded to the buyer with the highest valuation at the low price $p_{l}$. Since the other buyers cannot afford the remaining good at $p_{h}$ the expected profit of the seller is:

$$
\int_{p_{h}}^{1} \int_{p_{l}}^{p_{h}} \int_{p_{l}}^{x} p_{l} d y d x d v
$$

Note, that integrals (c) and (d) may be summed up.

e) Finally, notice that for all $v>p_{h}$ and $x>p_{h}$ irrespectively of where $y$ is realized, seller will be able to sell both goods and earn $p_{l}+p_{h}$ : 


$$
\int_{p_{h}}^{1} \int_{p_{h}}^{v} \int_{0}^{x}\left(p_{l}+p_{h}\right) d y d x d v
$$

Hence, the expected seller's profit is the summation of all integrals above accounting for corresponding joint densities:

$$
\begin{gathered}
\pi^{P P}=6\left[\int_{p_{l}}^{p_{h}} \int_{0}^{v} \int_{0}^{x} p_{l} d y d x d v+\int_{p_{h}}^{1} \int_{0}^{p_{l}} \int_{0}^{x} p_{l} d y d x d v+\right. \\
\left.\int_{p_{h}}^{1} \int_{p_{l}}^{p_{h}} \int_{0}^{x} p_{l} d y d x d v+\int_{p_{h}}^{1} \int_{p_{h}}^{v} \int_{0}^{x}\left(p_{l}+p_{h}\right) d y d x d v\right]= \\
=p_{h}-3 p_{h}^{3}+2 p_{h}^{4}+p_{l}-p_{l}^{4}
\end{gathered}
$$

From the profit function it is seen that $p_{l}$ and $p_{h}$ do not have an effect on each other. Hence, the profit function may be decomposed into two streams: profit received from the low price $\pi_{p_{l}}$ and profit received from the high price $\pi_{p_{h}}$. Figure 2.2 presents a graphical illustration of both streams.

To find the price which maximizes overall profit of the seller it is first helpful to identify the low price at which corresponding profit stream is maximized, which happens at $\frac{\partial \pi^{P P}}{\partial p_{l}}=0$ or when $p_{l}=0.630$. The shape of the profit stream from the high price calls for a corner solution at $p_{h}=0.630+\epsilon$ since $p_{l}<p_{h}$. However, the seller can do better. Notice that the slopes of two profit functions in the vicinity of $p=0.630$ are different and the marginal loss from decreasing $p_{l}$ is lower than the marginal gain from decreasing $p_{h}$. Therefore, I need to find such a price, at which the slopes of two profit streams coincide, which can be done by solving the following equation: 


$$
\frac{\partial \pi^{P P}}{\partial p_{l}}=\frac{\partial \pi^{P P}}{\partial p_{h}}, \text { or } 4 p^{3}-1=1+8 p^{3}-9 p^{2}
$$

Hence, marginal loss from decreasing $p_{l}$ is equal to the marginal gain from decreasing $p_{h}$ when $p^{*}=p_{l}=p_{h}=0.541$. This is the price the seller uses in the equilibrium and earns a profit of $\pi^{P P}=0.692^{8}$.

Figure 2.2: Two streams of profit from simultaneously using posted prices - low and high.

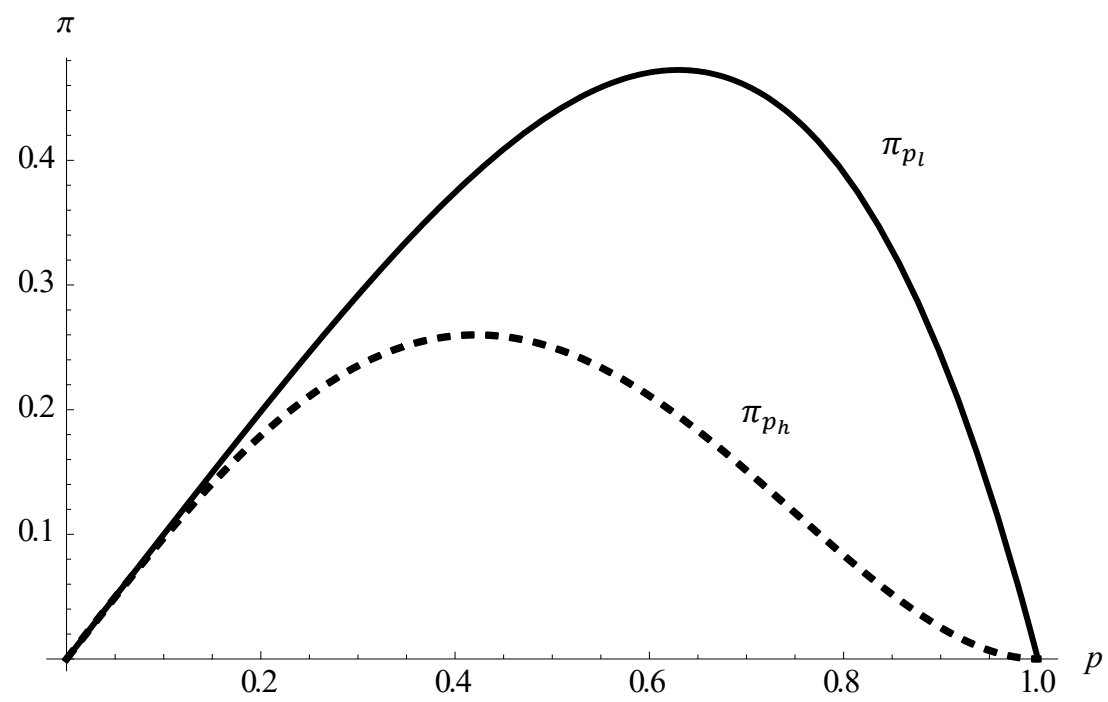

Remark. The results above do not change significantly even without the assumption 1, i.e. if buyers are awarded the good randomly with equal probability. In this case integral (c) becomes $\int_{p_{h}}^{1} \int_{p_{l}}^{p_{h}} \int_{0}^{p_{l}}\left(p_{l}+\frac{1}{2} p_{h}\right) d y d x d v$ and integral (d) -

\footnotetext{
${ }^{8}$ Assuming that the seller uses the same prices to sell his goods it would be easy to formulate the objective function. If all buyers' valuations are below the posted price then the seller receives no profit. If one of the buyer's valuations is higher than the posted price then the seller will sell one good to this buyer and receive $p$. If two and more buyers have valuations higher than the posted price then the seller's profit will be equal to $2 p: \pi^{P P}=6\left(\int_{p}^{1} \int_{0}^{p} \int_{0}^{x} p d y d x d v+\int_{p}^{1} \int_{p}^{v} \int_{0}^{x} 2 p d y d x d v\right)=$ $3\left(p^{3}-p^{4}\right)+2 p-6 p^{3}+4 p^{4}=2 p-3 p^{3}+p^{4}$ The profit is maximized at $p=0.541$ and the seller earns 0.692 , which matches the numbers in the proof.
} 
$\int_{p_{h}}^{1} \int_{p_{l}}^{p_{h}} \int_{p_{l}}^{x}\left(p_{l}+\frac{2}{3} p_{h}\right) d y d x d v$. Hence, the whole maximization problem transforms into:

$$
\begin{gathered}
6\left[\int_{p_{l}}^{p_{h}} \int_{0}^{v} \int_{0}^{x} p_{l} d y d x d v+\int_{p_{h}}^{1} \int_{0}^{p_{l}} \int_{0}^{x} p_{l} d y d x d v+\right. \\
\int_{p_{h}}^{1} \int_{p_{l}}^{p_{h}} \int_{0}^{p_{l}}\left(p_{l}+\frac{1}{2} p_{h}\right) d y d x d v+\int_{p_{h}}^{1} \int_{p_{l}}^{p_{h}} \int_{p_{l}}^{x}\left(p_{l}+\frac{2}{3} p_{h}\right) d y d x d v+ \\
\left.\int_{p_{h}}^{1} \int_{p_{h}}^{v} \int_{0}^{x}\left(p_{l}+p_{h}\right) d y d x d v\right]=\left(1-p_{l}\right)\left(p_{l}\left(1+p_{l}+p_{l}^{2}\right)+p_{h}\left(1+p_{l}\right)-p_{h}^{2} p_{l}-p_{h}^{3}\right)
\end{gathered}
$$

FOCs with respect to prices yield:

$$
\left\{\begin{array}{l}
\frac{\partial \pi^{P P}}{p_{l}}=1-4 p_{l}^{3}+\left(p_{h}-1\right)\left(p_{h}+2 p_{l}\right) p_{h}=0 \\
\frac{\partial \pi^{P P}}{p_{h}}=\left(1-p_{l}\right)\left(1+p_{l}-3 p_{h}^{2}-2 p_{h} p_{l}\right)=0
\end{array}\right.
$$

Solving the system of equations above produces the following profit-maximizing prices: $p_{l}=0.531$ and $p_{h}=0.559$.

\subsubsection{Auction - auction}

When a seller sells both goods simultaneously by auctions the payoff depends on the "rules of the game", i.e. what type of auctions are used, how bidders make their bids etc... My auction setup is in some spirit similar to the one examined by Peters and Severinov (2006). They consider simultaneous use of auctions in Internet sales with multiple increment bidding on a grid of valuations.

Proposition 6. The expected revenue of the monopolistic seller from using auctions to simultaneously sell two goods to three buyers whose valuations are dis- 
tributed uniformly is equal to 0.5 .

Proof. The assumption 1 in the setup of my model suggests that the buyer with the highest valuation is the first to choose between the auctions. Then, when the second-highest valuation buyer makes his choice he opts for another auction since the expected payoff is obviously higher when there are no competitors in the auction. In addition, he knows that the first buyer has higher valuation since the assumed rationing rule is common knowledge. Finally, when the third buyer arrives, it does not make a difference whether he bids sequentially or simultaneously in both auctions. He bids up to his valuation and loses. Hence, both auctions end at the valuation of the third buyer:

$$
\pi^{A A}=6\left(\int_{0}^{1} \int_{0}^{v} \int_{0}^{x} 2 y d y d x d v\right)=0.5
$$

\subsubsection{Discussion}

In this section I have analyzed the seller's revenue from selling two homogeneous goods to three buyers with uniformly distributed valuations. I have examined three possible selling mechanisms - auctions only, posted prices only and auction and a posted price.

Theorem 1. Under monopoly, given that there are three risk-neutral buyers with uniformly distributed valuations, it is optimal to sell two goods at posted prices.

Proof. The expected revenues of the monopolistic seller from using different mechanisms are ranked as follows (which follows directly from Propositions 4-6): 
Table 2.1: Comparing seller's revenues

\begin{tabular}{c|c|c} 
Posted Price & Posted Price & 0.692 \\
Auction & Posted Price & 0.680 \\
Auction & Auction & 0.500
\end{tabular}

From the table 2.1 it is seen that the seller's profit from simultaneously using posted prices exceeds the profit gained from the other mechanisms. Further I will show that this result does not hold when there is competition between sellers and in situations when the number of buyers becomes large or their risk aversion increases. However, this particular case is of interest since it tells us that if buyers are risk neutral and the difference between their number and the amount of goods in the market is small, posted prices is the best response for monopolistic sellers. On top of purely rational reasons it has been argued by Einav et al. $(2016)^{9}$ that eBay sellers choose a psychologically more convenient mechanism of posted price selling ${ }^{10}$. Nowadays, sellers rarely use simultaneous auctions only to sell their goods, but rather either auctions and posted prices or posted prices only. Also, an experienced seller is more likely to make rational decisions (List (2004)); in this case it means gradual shifting from simultaneous use of auctions to a mixed mechanism or posted prices. The presence of the latter phenomenon was also documented by Einav et al. (2016). In addition, in the last years there has been an influx of sellers to online platforms,

\footnotetext{
${ }^{9}$ The authors did not specifically analyze the market of finite goods, so my results corroborate at least a part of the observed phenomena.

${ }^{10}$ It is also possible that the transaction costs associated with holding an auction are higher than those for a posted price.
} 
which has made the environment of the latter similar to the one examined in this manuscript.

Finally, in auctions with a BIN price, if buyers are risk neutral, implementing BIN price does not increase sellers' profits in comparison to a standard second-price auction (Reynolds and Wooders (2009)). However, the authors do not compare it with price posting. In the examined baseline case of my model auctions generate less revenue than the mixed mechanism and even more so with simultaneous posted price selling when buyers are risk neutral.

\subsection{Two sellers (duopoly)}

Consider the same model as discussed so far, but now each homogeneous object is offered by a different seller, and they move sequentially. It is unlikely that in reality sellers would post their goods simultaneously. A more realistic scenario is when one seller puts his object for sale and another seller, after observing the move of the first seller, chooses his action. Buyers arrive to the market after both objects were posted (in other words, the first seller cannot act as a monopolist before the arrival of the second seller). Hence, each seller has the following set of strategies: $p \in[0,1] \cup a$, where $a$ - auction. Since the action space for $p$ is continuous on $[0,1]$ I will first find best response functions for three classes of strategy profiles $(a, a),(a, p)$ and $(p, p)$. Then, I will check which of them provides the highest revenues. 


\subsubsection{Auction - posted price}

Consider a case when the first seller uses auction and the other seller replies with a posted price. To find the best response function of the seller choosing a price to the other seller choosing an auction I need to decompose the profit function from the monopoly case into two streams of revenues - profit from the auction and profit from the posted price $\left(\pi_{A}\left(\pi_{P}\right)\right.$ is the profit from using the auction (posted price) when another seller chooses posted price (auction)).

Lemma 7. When one seller uses an auction and the other seller uses a posted price, or vice versa, their profits are defined by the following functions:

$$
\begin{gathered}
{\left[\begin{array}{l}
\pi_{A}=-\frac{71}{256}+\frac{135 p}{64}-\frac{213 p^{2}}{128}+\frac{7 p^{3}}{64}+\frac{57 p^{4}}{256} \quad \text { if } p>\frac{3}{7} \\
\pi_{P}=\frac{25 p}{16}-\frac{27 p^{2}}{16}-\frac{21 p^{3}}{16}+\frac{23 p^{4}}{16} \\
{\left[\begin{array}{l}
\pi_{A}=\frac{1}{4}+\frac{31 p^{4}}{12} \\
\pi_{P}=p-\frac{11 p^{4}}{4}
\end{array}\right.}
\end{array} \quad \text { if } p \leq \frac{3}{7}\right.}
\end{gathered}
$$

Proof. Recall that the profit function of the monopolistic seller is described by two functions for each domain of $p$. Hence, the decomposition results into: 


$$
\begin{aligned}
& \pi_{A} \mid p \leq \frac{3}{7}=6\left[\int_{0}^{p} \int_{0}^{v} \int_{0}^{x} x d y d x d v+\int_{p}^{\frac{7}{3} p} \int_{0}^{\frac{7}{4} p-\frac{3}{4} v} \int_{0}^{x} x d y d x d v+\right. \\
& \int_{p}^{\frac{7}{3} p} \int_{\frac{7}{4} p-\frac{3}{4} v}^{p} \int_{0}^{\frac{7}{4} p-\frac{3}{4} v} x d y d x d v+\int_{p}^{\frac{7}{3} p} \int_{\frac{7}{4} p-\frac{3}{4} v}^{p} \int_{\frac{7}{4} p-\frac{3}{4} v}^{x} y d y d x d v+ \\
& \int_{p}^{\frac{7}{3} p} \int_{p}^{v} \int_{0}^{\frac{7}{4} p-\frac{3}{4} v} p d y d x d v+\int_{p}^{\frac{7}{3} p} \int_{p}^{v} \int_{\frac{7}{4} p-\frac{3}{4} v}^{p} y d y d x d v+ \\
& \left.\int_{p}^{\frac{7}{3} p} \int_{p}^{v} \int_{p}^{x} y p d y d x d v+\int_{\frac{7}{3} p}^{1} \int_{0}^{v} \int_{0}^{x} y d y d x d v\right]= \\
& 6\left[\frac{p^{4}}{12}+\frac{p^{4}}{9}+\frac{p^{4}}{6}+\frac{p^{4}}{9}+\frac{8 p^{4}}{27}+\frac{10 p^{4}}{27}+\frac{128 p^{4}}{243}+\frac{81-2401 p^{4}}{1944}\right] \\
& \pi_{P} \mid p \leq \frac{3}{7}=6\left[\int_{p}^{\frac{7}{3} p} \int_{\frac{7}{4} p-\frac{3}{4} v}^{p} \int_{\frac{7}{4} p-\frac{3}{4} v}^{x} p d y d x d v+\right. \\
& \int_{p}^{\frac{7}{3} p} \int_{p}^{v} \int_{0}^{\frac{7}{4} p-\frac{3}{4} v} p d y d x d v+\int_{p}^{\frac{7}{3} p} \int_{p}^{v} \int_{\frac{7}{4} p-\frac{3}{4} v}^{p} p d y d x d v+ \\
& \left.\int_{p}^{\frac{7}{3} p} \int_{p}^{v} \int_{p}^{x} p p d y d x d v+\int_{\frac{7}{3} p}^{1} \int_{0}^{v} \int_{0}^{x} p d y d x d v\right]= \\
& 6\left[\frac{2 p^{4}}{9}+\frac{8 p^{4}}{27}+\frac{16 p^{4}}{27}+\frac{32 p^{4}}{81}+\frac{27 p-343 p^{4}}{162}\right] \\
& \pi_{A} \mid p>\frac{3}{7}=6\left[\int_{0}^{p} \int_{0}^{v} \int_{0}^{x} x d y d x d v+\int_{p}^{1} \int_{0}^{\frac{7}{4} p-\frac{3}{4} v} \int_{0}^{x} x d y d x d v+\right. \\
& \int_{p}^{1} \int_{\frac{7}{4} p-\frac{3}{4} v}^{p} \int_{0}^{\frac{7}{4} p-\frac{3}{4} v} x d y d x d v+\int_{p}^{1} \int_{\frac{7}{4} p-\frac{3}{4} v}^{p} \int_{\frac{7}{4} p-\frac{3}{4} v}^{x} y d y d x d v+ \\
& \int_{p}^{1} \int_{p}^{v} \int_{0}^{\frac{7}{4} p-\frac{3}{4} v} p d y d x d v+\int_{p}^{1} \int_{p}^{v} \int_{\frac{7}{4} p-\frac{3}{4} v}^{p} y d y d x d v+ \\
& \left.\int_{p}^{1} \int_{p}^{v} \int_{p}^{x} y d y d x d v\right]=-\frac{71}{256}+\frac{135 p}{64}-\frac{213 p^{2}}{128}+\frac{7 p^{3}}{64}+\frac{57 p^{4}}{256}
\end{aligned}
$$




$$
\begin{gathered}
\pi_{P} \mid p>\frac{3}{7}=6\left[\int_{p}^{1} \int_{\frac{7}{4} p-\frac{3}{4} v}^{p} \int_{\frac{7}{4} p-\frac{3}{4} v}^{x} p d y d x d v+\right. \\
\int_{p}^{1} \int_{p}^{v} \int_{0}^{\frac{7}{4} p-\frac{3}{4} v} p d y d x d v+\int_{p}^{1} \int_{p}^{v} \int_{\frac{7}{4} p-\frac{3}{4} v}^{p} p d y d x d v+ \\
\left.\int_{p}^{1} \int_{p}^{v} \int_{p}^{x} p d y d x d v\right]=\frac{25 p}{16}-\frac{27 p^{2}}{16}-\frac{21 p^{3}}{16}+\frac{23 p^{4}}{16}
\end{gathered}
$$

Simplifying, produces the result for each identified domain of $p$ (figure 2.3 provides a graphical illustration).

Figure 2.3: Decomposed sellers' profits from simultaneously using an auction and a posted price.

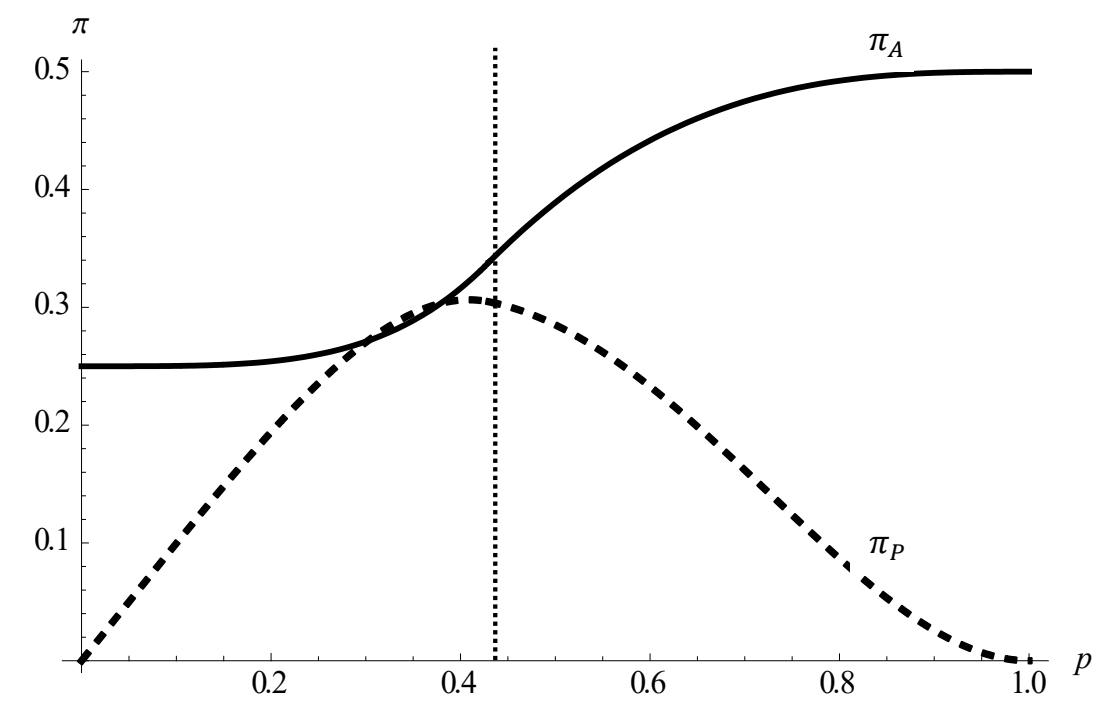

Notice that If there was only one seller or two sellers acting cooperatively, the equations in Lemma 7 could be summed up and maximized with respect to $p$, which was done for the case of a single seller before. 


\subsubsection{Auction - auction}

I assumed that sellers do not choose reserve prices. However, the literature shows that competition in auctions drives reserve price to marginal $\operatorname{costs}^{11}$. Thus, the equilibrium is exactly the same as in the monopoly case. This time, however, one seller gets only half of the cumulative profit, i.e. $\pi_{A A}^{i}=0.25$, where $i=1,2$.

\subsubsection{Posted price - posted price}

When two sellers compete in prices they play a game similar to a Bertrand competition with homogeneous products and capacity constraints. A detailed exposition of this game can be found in, for example, Levitan and Shubik (1972) or Kreps and Scheinkman (1983). However, my model is different in three distinct ways. First, sellers do not choose capacity - it is fixed. Second, the demand is stochastic and discontinuous ${ }^{12}$. Third, players choose prices sequentially. If the sellers were choosing prices simultaneously then the equilibrium of the game would look somewhat similar to the equilibrium in Edgeworth-Bertrand models: there would exist mixed equilibrium on the support of prices known as Edgeworth Cycle (Edgeworth (1897)). However, the infimum of the corresponding profits would be higher if sellers were competing in both posted prices and auctions rather than posted prices only ${ }^{13}$.

To understand how the equilibrium will look like in the sequential game I need

\footnotetext{
${ }^{11}$ However, Burguet and Sákovics (1999) show that it may not always be the case for duopoly when sellers compete in second-price sealed bid auctions.

${ }^{12}$ Sellers know only the distribution of buyers' valuations. Hence, for different prices, conditional on the realized distribution, they may face different demand. Since there are only 3 buyers the demand is discontinuous at each price.

${ }^{13}$ Due to the complicated nature of this analysis it is not included here, but available on request.
} 
to return to the decomposed profit function from the case of one seller ${ }^{14}$. For a low price the profit may be written as follows:

$$
\pi_{p_{l}}=6 \int_{p_{l}}^{1} \int_{0}^{v} \int_{0}^{x} p_{l} d y d x d v=p_{l}-p_{l}^{4}
$$

For a high price the profit of the seller is defined as:

$$
\pi_{p_{h}}=6 \int_{p_{h}}^{1} \int_{p_{h}}^{v} \int_{0}^{x} p_{h} d y d x d v=p_{h}-3 p_{h}^{3}+2 p_{h}^{4}
$$

\subsubsection{Best responses}

\subsubsection{First seller chooses auction}

Proposition 7. When the first seller chooses an auction the best response of the second seller is to choose an optimal posted price.

Proof. If the first-moving seller chooses an auction the other seller may reply with either an auction or any posted price $p \in[0,1]$. If he replies with the auction his profit is $\pi=0.25$. If the latter seller replies with the posted price then both sellers' revenues are defined by profit functions presented on figure 2.3. The best response of the seller replying with the posted price is to set it at the level, which maximizes his stream of profits, and the other seller will take it as given. In other words, the profit of the seller who uses the posted price can be found by maximizing his profit stream $\pi_{P}$ with respect to $p$. The profit of the seller who uses the auction can be found by substituting the profit-maximizing posted price of the $\pi_{P}$ profit stream into the $\pi_{A}$ :

\footnotetext{
${ }^{14}$ Recall that $p_{l}$ is the low price and $p_{h}$ is the high price.
} 


$$
\begin{gathered}
\frac{d \pi_{P}}{d p}=1-\frac{44 p^{3}}{3}=0, \text { where } p \in\left[0, \frac{3}{7}\right] \\
\frac{d \pi_{P}}{d p}=\frac{25}{16}-\frac{27 p}{8}-\frac{63 p^{2}}{16}+\frac{23 p^{3}}{4}=0, \text { where } p \in\left(\frac{3}{7}, 1\right]
\end{gathered}
$$

Equation (2.22) holds when $p=0.409$; equation $(2.23)$ - when $p=0.387$, which is outside the defined range for $p$. Hence, $p^{*}=0.409$ is the price chosen by one seller as a response to the other seller choosing an auction. Substituting it into the corresponding profit functions (i.e. for $p \leq \frac{3}{7}$ ) of both sellers I get:

$$
\begin{gathered}
\pi_{A}\left(p^{*}\right)=\frac{1}{4}+\frac{31 \times(0.409)^{4}}{12}=0.322 \\
\pi_{P}\left(p^{*}\right)=0.409-\frac{11 \times(0.409)^{4}}{4}=0.306
\end{gathered}
$$

where $\pi_{A}$ is the profit of the seller who uses the auction, $\pi_{P}$ - profit of the seller who uses the posted price. Since $0.306>0.250$ the second seller will choose an optimal price.

Thus, if the first seller chooses an auction, it is the best response for the other seller to reply with an optimal posted price and both sellers earn profits higher than 0.3 .

\subsubsection{First seller chooses price}

Proposition 8. When the first seller chooses a posted price it is the best response of the other seller to reply with either an auction or a posted price conditional on the level of the posted price chosen by the first seller. 
Proof. The seller can choose any price $p \in[0,1]$, and it can be replied with either an auction or any price from the same domain by the other seller, which results in a continuum of payoffs. However, I will show that there are several regions of the price, for which the response of the other seller remains the same. Figure 2.4 is helpful in analyzing this game. It presents profit functions describing the seller's revenues when he replies with either price (depending on whether it is higher or lower than the price of the other seller) or auction to the other seller choosing a price.

First, note that a seller has no incentive to reply with a higher price unless the price named by the first-moving seller is below the price making the former seller indifferent between undercutting and setting a maximizing price for the profit from the high price. The profit of the seller selling at the high price is maximized when $\frac{d \pi_{p_{h}}}{d p}=0$ or when $p=0.422$ and is equal to $\pi_{p_{h}}=0.260$. Hence, $\pi_{p_{l}}=p_{l}-p_{l}^{4}=0.260 \Rightarrow p=$ 0.265 . However, at some of these prices another seller is better off replying with an auction. To find this range of prices set the profit function from the auction equal to the maximum of the profit gained from the high price, i.e. $\frac{1}{4}+\frac{31 p^{4}}{12}=0.260$, which gives the price $p=0.249$ (point A on figure 2.4). Hence, when the first-moving seller names any price $p \in[0,0.249]$ the best response of the other seller is to set his price equal to $p=0.422$; if the former price is $p \in[0.249,0.265]$, the best response of the other seller is to use the auction. 
Figure 2.4: The continuum of payoffs for sellers using mixed mechanism or posted prices only.

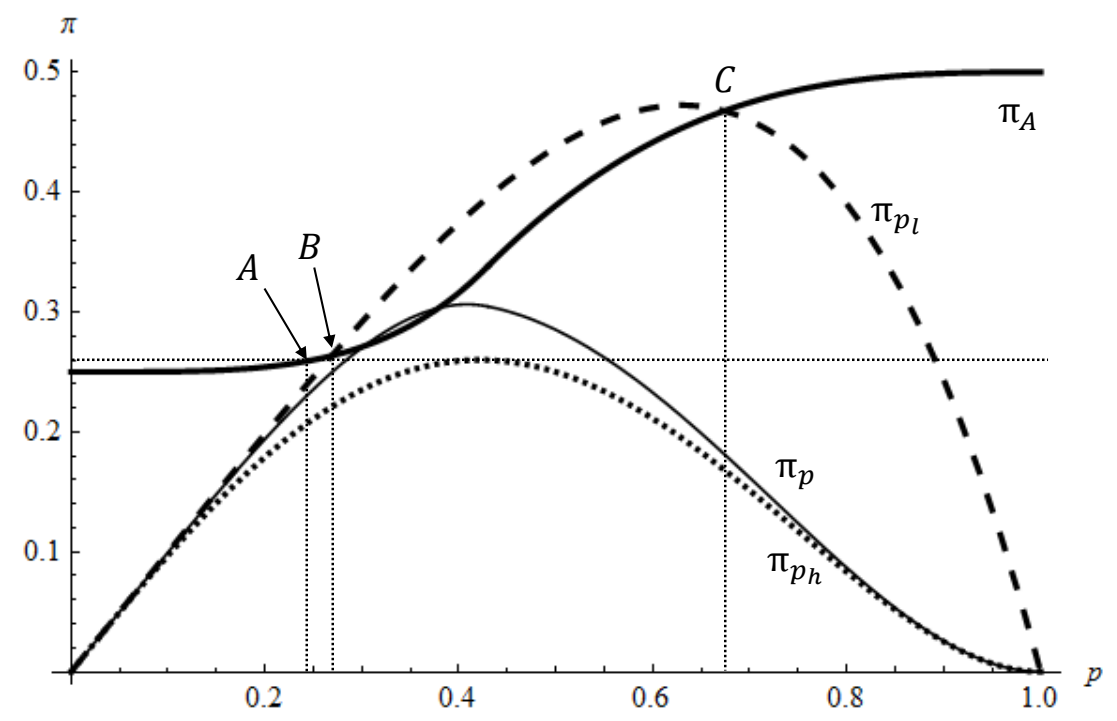

Second, if there was no auction available the best response of the seller for any price $p>0.265$ would be to undercut. However, when replying with an auction is a possibility, there is a range of prices for which it is more profitable to reply with an auction rather than to undercut. This range could be found by setting profit from the auction equal to the profit from the low price ${ }^{15}$.

$$
\begin{gathered}
\pi_{A}=\pi_{p_{l}} \Rightarrow \frac{1}{4}+\frac{31 p^{4}}{12}=p-p^{4}, \text { if } p \leq \frac{3}{7} \\
\pi_{A}=\pi_{p_{l}} \Rightarrow-\frac{71}{256}+\frac{135 p}{64}-\frac{213 p^{2}}{128}+\frac{7 p^{3}}{64}+\frac{57 p^{4}}{256}=p-p^{4}, \text { if } p>\frac{3}{7}
\end{gathered}
$$

The equations above hold when $p=0.269$ and $p=0.674$ (points $\mathrm{B}$ and $\mathrm{C}$

\footnotetext{
${ }^{15}$ Notice that the higher region intersects the profit function from the auction when $p>\frac{3}{7}$ and the lower — when $p<\frac{3}{7}$.
} 
respectively on figure 2.4). If $p>0.674$ and $0.249<p<0.269$ then it is the best response of the other seller to use the auction. When $0.269 \leq p \leq 0.647$ the seller will undercut. Lastly, when $p<0.249$ the other seller sets the price $p=0.422$.

\subsubsection{Discussion}

Previously, I found best responses for each action that the first-moving seller chooses. They are summarized in the following theorem:

Theorem 2. Under duopoly, given that there are three buyers with uniformly distributed valuations, it is optimal of the first-moving seller to offer his good by an auction. The second seller replies with a profit-maximizing price.

Proof. The proof follows directly from Propositions 7-8. To simplify things I provide only the maximum profit from a corresponding region the first seller gets since the direction of the game is decided by the seller who moves first. The continuum of payoffs for both players is defined by $\pi_{A}, \pi_{P}, \pi_{p_{l}}$ and $\pi_{p_{h}}$, which are displayed on figure 2.4 . 
Table 2.2: Sellers' best responses

\begin{tabular}{c|c|c} 
Seller 1 & Seller 2 & Max profit of Seller 1 \\
\hline Auction & $p=0.403$ & 0.322 \\
$0 \leq p<0.249$ & $p=0.422$ & 0.245 \\
$0.249 \leq p<0.269$ & Auction & 0.250 \\
$0.269 \leq p<0.674$ & $p-\epsilon$ & 0.260 \\
$0.674 \leq p \leq 1$ & Auction & 0.201 \\
\hline
\end{tabular}

If auction is the response of at least one of the sellers then their profits are defined by functions $\pi_{A}$ and $\pi_{P}$. If only posted prices are used as best responses then the profits are defined by $\pi_{p_{l}}$ and $\pi_{p_{h}}$. Hence, the four functions account for all possible off-equilibrium paths.

Table 2 shows that the highest revenue the first seller can receive when moving first is by using an auction. The other seller replies with an optimal posted price, which results into the formation of a mixed mechanism on the market. Of course, the other seller could earn more by switching to an auction if the first seller named a very high price or undercut if the price was in the middle range. However, foreseeing this, the first seller uses the mechanism which hedges him against such actions from the other seller and compels the latter to reply with an optimal posted price.

Finally, if using auctions was not possible on the market then the first seller would choose a price $\left(p_{l}=0.265\right)$ which earns him the same profit as the maximum of 
the seller's profit who offers his good at the high price (which occurs at $\left.p_{h}=0.422\right)^{16}$. In this case both sellers would earn $\pi=0.26$, which is lower than the profits they get under the mixed mechanism. Since buyers are risk neutral it directly follows that they are worse under the mixed mechanism rather than competition in posted prices only.

${ }^{16}$ It is easy to check that this is the only equilibrium in posted prices. 


\section{Chapter 3}

\section{Extensions}

In this chapter I provide several extensions to the equilibrium of the buyers' subgame and show how these changes affect the revenues of the monopolistic seller.

\subsection{Two objects and $n$ buyers}

\subsubsection{General case}

To extend the model to $n$ buyers I need to make a simplifying assumption that bidders still retain two rounds of bidding. In the model with 3 buyers I have discussed that the number of rounds of bidding may not be higher than $n-1$, which was 2. Imagine that a bidder commits to some exit price given the number of active bidders he observes in the auction. In the model with 3 buyers, when the auction price reaches the exit price of a bidder, there are only three possibilities: either one of the other bidders drops out, one of the other bidders exits and buys the good at the posted price or both other bidders remain in the auction. I showed that, conditional 
on the change of this information at the exit price level, the bidder would either stay or leave the auction. With $n$ bidders there are potentially $n-1$ rounds of bidding, and the strategy quickly becomes intractable. For example, in the beginning of the auction a bidder commits to a certain exit price given, say, five bidders in the auction. At this price he observes that there are four bidders left and the posted price is still available signaling that the fifth bidder has dropped out from the auction. Hence, the first bidder increases his new exit price based on this information. Then, at the new exit price one additional bidder drops out, and the bidder in question again needs to increase his exit price for the case of three bidders. If the number of bidders is large, there are potentially many exit prices which would be selected at each bidding period. I will trim its space by assuming that the bidder still has two rounds of bidding, and he changes his decision to leave the auction only when there are 2 players left in the auction at the first exit price he has committed to in the beginning of the auction ${ }^{1}$.

To derive the expected payoff I need to use the joint density between the second highest valuation and the order statistics of the maximum of the third highest valuation because the domains of integration differ across the payoff structure. Therefore, the joint density could be written as $(n-1) f(x)(n-2) F(y)^{n-3} f(y)$, where $y$ is the maximum of the order statistics of the $n-2$ players, which turns to just $2 f(x) f(y)$ for the case of 3 players. By changing joint densities in the equation (1.3) I can calculate the expected payoff. The result is captured by the following lemma:

\footnotetext{
${ }^{1}$ Note that as $n$ increases the equilibrium of the game becomes similar to the case when a bidder is not able to observe the number of bidders. The latter scenario is not used due to intractability.
} 
Lemma 8. The derivative of the equilibrium function mapping buyer's valuation into the exit price for all $v>p$ when there are $n$ players is described by the following equation:

$$
z^{\prime}(v)=\theta \frac{\left.(v-p)\left(F(c)^{n-2}-F(v)^{n-2}\right)+(n-2) \int_{c}^{v}(v-y) F(y)^{n-3} f(y) d y\right)}{F(c)(c-p)+\int_{c}^{p} F(x) d x}
$$

where $\theta=\frac{f(v)}{(n-2) f(c) F(c)^{n-3}}>0$ and the function has the following properties:

a) The function $z(v)$ exists only if $c<p$.

b) The function $z(v)$ is negative only if $v-p>E(v-y)$ on $[c, v]$, where $y$ is the maximum of the order statistics of $n-2$.

c) The slope of the function $z(v)$ increases when the posted price decreases and vice versa.

d) The slope of the function $z(v)$ increases when the number of bidders increases and vice versa.

\section{Proof. Appendix D.}

Lemma 8 shows that the equilibrium function in the $n$-buyers case is similar to the one in the $3 \times 2$ case. This time, however, the number of buyers also plays a role in determining its slope, and, as a corollary, there is a set of prices and the number of players for which the equilibrium function will not have a kink at the point $z(v)=0^{2}$, while for the other combinations - it will. The following proposition states the equilibrium for the $n$-bidders:

\footnotetext{
${ }^{2}$ With assumption 1 still holding.
} 
Proposition 9. There is an equilibrium in which:

$$
\beta(v, p)= \begin{cases}v & \text { if } v \leq p \\ z(v) \text { with } z^{\prime}(v) \text { defined by }(3.1) & \text { if } v>p, p>\tilde{p}(n) \\ z(v) \text { with } z^{\prime}(v) \text { defined by }(3.1) & \text { if } p<v<\hat{v}, p \leq \tilde{p}(n) \\ 0 & \text { if } \hat{v} \leq v \leq \bar{v}, p \leq \tilde{p}(n)\end{cases}
$$

Where beliefs are determined by $\beta$ and $\tilde{p}(n)$ is the combination of all possible numbers of players and posted prices at which $z(v)=0$ and $v=1$.

\subsubsection{Uniform distribution}

By plugging corresponding PDFs $(f(\psi)=1)$ and $\operatorname{CDFs}(F(\psi)=\psi)$ into the equation (3.1) and some rearrangements I receive the following equation:

$$
\frac{1}{2}(p-z(v))^{2} z(v)^{n-3} z^{\prime}(v)=\frac{v^{n-2}-z(v)^{n-2}}{n-2} p-\frac{v^{n-1}-z(v)^{n-1}}{n-1}
$$

Which could further be rewritten as a slope field:

$$
z^{\prime}(v)=-\frac{2\left(\frac{v^{n-1}-z(v)^{n-1}}{n-1}-\frac{v^{n-2}-z(v)^{n-2}}{n-2} p\right)}{z(v)^{n-3}(p-z(v))^{2}}
$$

The differential equations above do not have a closed-form solution, but I implement local analysis and present an approximate solution by perturbing and expanding the function around the point $[p, p]$ : 
Lemma 9. When there are $n$ buyers the exit function is described by the following equation:

$$
z(v)=\left(\frac{7}{4}+r\right) p-\left(\frac{3}{4}+2 r\right) v+\frac{r v^{2}}{p}
$$

where $r=1.012-0.3373 n$

Proof. Appendix E.

When there are many buyers in the market, given the analytical form of their reaction function, it is readily seen that at some valuation (less than 1) the exit function will be equal to zero. All buyers with valuations above will prefer to buy the good at the posted price before the auction starts. To find this point set $z(v)=0$ and express $v$ as a function of $p$ and $n$ :

$$
\hat{v}=\frac{p(3+8 r-\sqrt{9-64 r})}{8 r}
$$

Recall from the $3 \times 2$ case that when $n=3$ and $p=\frac{3}{7}$ the exit function passes through the point $[1,0]$ (substituting $\hat{v}=1$ and $n=3$ into the equation (3.6) confirms it). By setting $\hat{v}=1$ in equation (3.6) I may also obtain a condition on $p$ and $n$ under which an indifferent bidder would exist for the case of $n$-bidders. The price as a function of the number of players may be expressed as follows:

$$
\tilde{p}=\frac{8 r}{3+8 r-\sqrt{9-64 r}}
$$

To further test the agreement between the resulting equation and the slope 
field I perform simulations for different values of $p$ and $n$ (figure 3.1, where solid line is the original equation, dashed line - approximating equation). Note that the isocurve is chosen based on the initial conditions $(p+\epsilon, p-\epsilon)$ and is robust to the value of $\epsilon$. The results show that with an increase in $n$ (and decrease in $p$ ) there is less agreement, which is expected from using only one perturbed term resulting in a quadratic function. The agreement would increase if additional perturbed terms were added, but in this particular case the complexity of the task would also rise exponentially.

Having tested how well the solution and conditions describe the original differential equation I am able to formulate the equilibrium for the $n$-bidders case when buyers' valuations are distributed uniformly. The equilibrium assumes a form similar to the $3 \mathrm{x} 2$ case except that now both the exit function (when $v>p$ ) and the existence of an indifferent bidder depend on the number of players in the auction:

Proposition 10. There is an equilibrium in which:

$$
\beta(v, p)= \begin{cases}v & \text { if } v \leq p \\ \left(\frac{7}{4}+r\right) p-\left(\frac{3}{4}+2 r\right) v+\frac{r v^{2}}{p} & \text { if } v>p, p>\tilde{p}(n) \\ \left(\frac{7}{4}+r\right) p-\left(\frac{3}{4}+2 r\right) v+\frac{r v^{2}}{p} & \text { if } p<v<\hat{v}, p \leq \tilde{p}(n) \\ 0 & \text { if } \hat{v} \leq v \leq \bar{v}, p \leq \tilde{p}(n)\end{cases}
$$

Where $\hat{v}$ is defined by (3.6), $\tilde{p}(n)$ is defined by $(3.7), r=1.012-0.3373 n^{3}$ and beliefs are determined by $\beta$.

\footnotetext{
${ }^{3}$ Note that when $n=3 r=0$ and the equilibrium becomes identical to the $3 \mathrm{x} 2$ case.
} 
Figure 3.1: Simulation of the degree of agreement between original equation and the approximate solution.
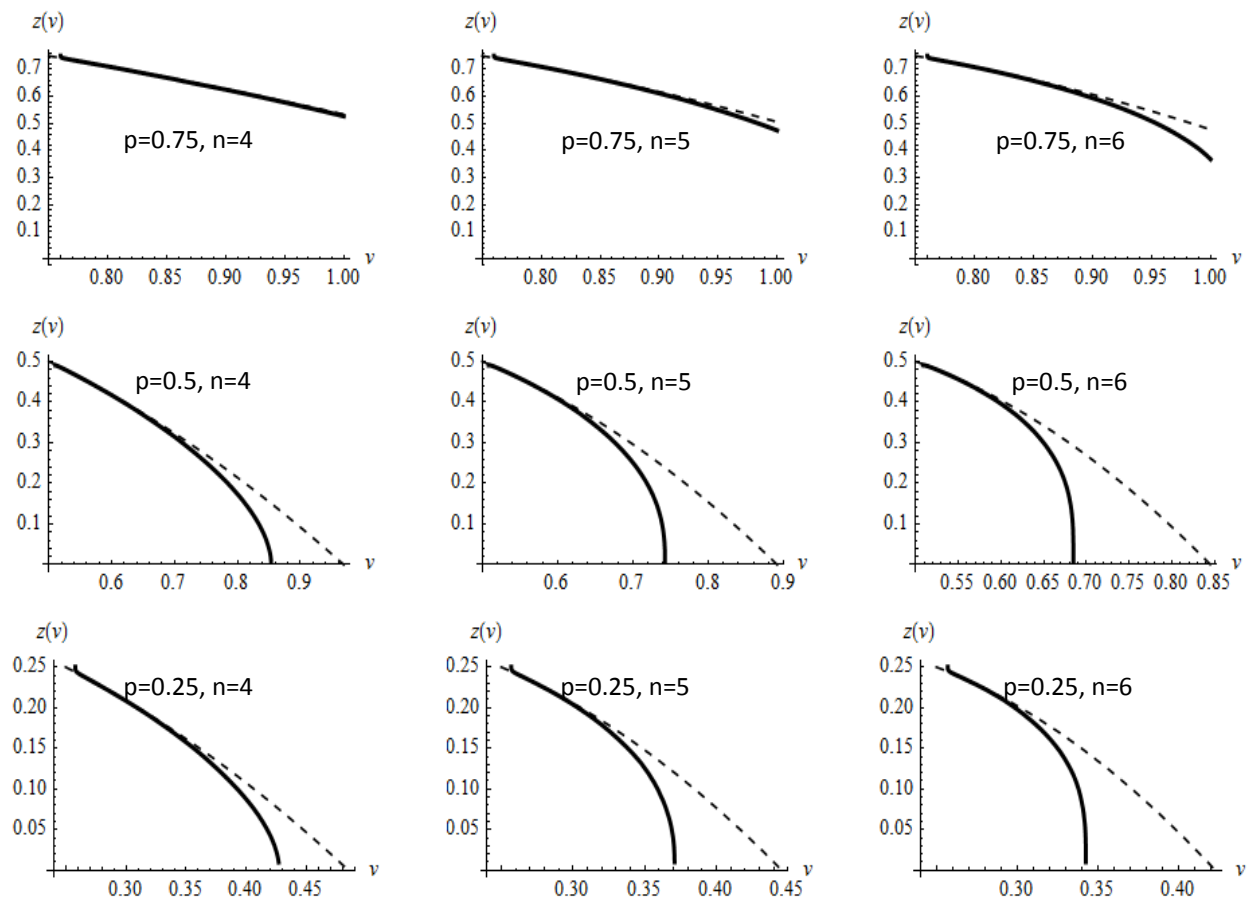

The incentives above represent an interesting feature of finite posted prices.

The probability of winning the auction at a price lower than the price outside decreases with the number of players who start in the auction and increases with the posted price itself. I do not consider the case of many sellers here, which would probably be intractable, but it is readily seen that the amount of goods available outside the auction will proportionally influence the probability of getting the good, and as a result - the incentives to exit earlier or later in the auction. When the number of goods is higher or equal to the number of buyers these incentives disappear since every buyer with a valuation higher than the posted price will for sure be able to get 
the good at most at the price equal to the posted price.

\subsubsection{Seller's profits}

With the equilibrium exit function for $n$ bidders I am able to further simulate profits of the seller from using different selling mechanisms ${ }^{4}$. The algorithm of simulations is the following. First, substitute different numbers of $n$ into the equation (3.5) to find the exit function; into the equation (3.6) to find an indifferent bidder; and into the equation (3.7) to find a threshold posted price for the existence of an indifferent bidder. Second, add an additional domain of integration which could be done either by adding an integral with a corresponding adjustment of the density, or order statistics of the density of the maximum of $n-2$ (the same is done for double auctions and double posted price). As a final step, run the maximization problem of the seller for new variables checking that the results satisfy the condition on $p$.

The results are presented on figure 3.2. As it turns out adding even one additional buyer makes the mixed mechanism more profitable than using posted prices only, and the difference persists with a further increase in the number of buyers. Also, when the amount of buyers becomes substantially larger than the number of goods, double auctions begin to produce higher profits than any other mechanism, even with the reservation price set to zero. These results reflect the regularities observed in reality, especially in the case of grand auction houses, selling unique goods to a large pool of buyers ${ }^{5}$.

\footnotetext{
${ }^{4}$ Analytical solution seems to be intractable.

${ }^{5}$ Note, however, that these are usually analyzed via a common-value framework.
} 
Figure 3.2: Simulation of profits from different mechanisms for different number of buyers.

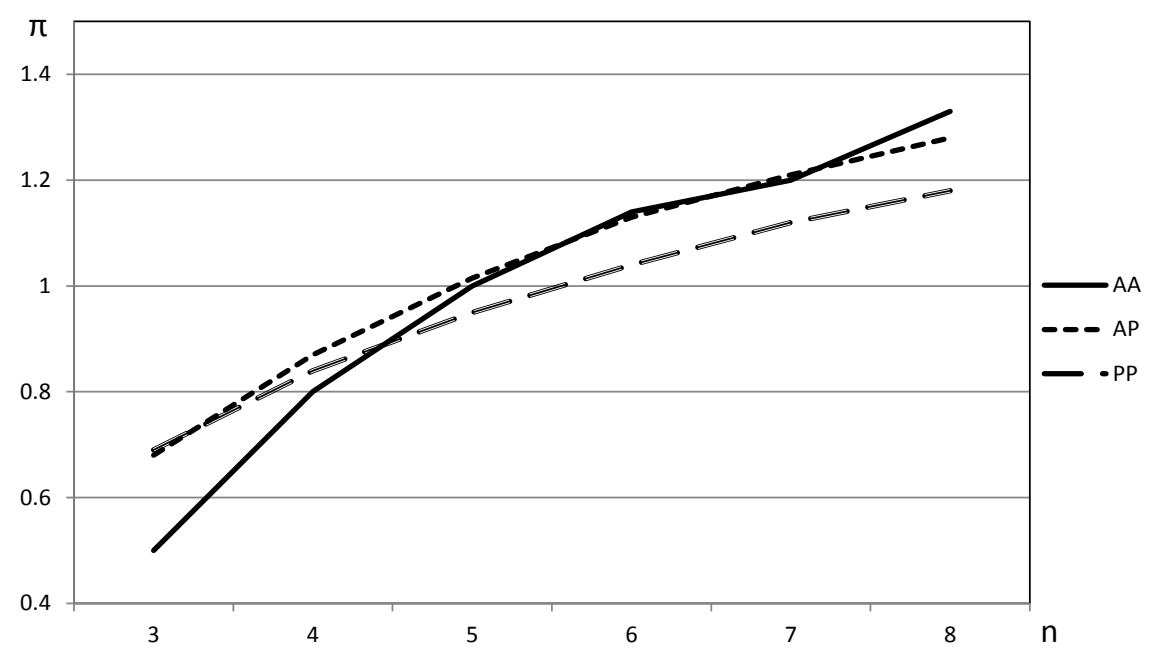

\subsection{Risk aversion}

\subsubsection{Buyers' equilibrium}

Risk aversion was shown to play a significant role in auctions with a BIN price (Reynolds and Wooders (2009); Chen et al. (2013); Anwar and Zheng (2015)). In fact, it was proved to be one of the major factors why such mechanism would be used in the first place. Intuitively, the more risk averse a buyer, the faster he will end the auction by accepting the BIN price, which in equilibrium is set with a premium. In this manuscript I did not introduce risk aversion to simplify the analysis and focus on the other aspects of bidding behavior. In what follows I will fill this gap and show how risk aversion could be introduced into the model of 3 buyers and 2 objects.

Following Chen et al. (2013) assume that the utility of the bidder $u(v)$ is defined 
as $\frac{u(v)^{\alpha}}{\alpha}$, with $0<\alpha \leq 1$ being the reverse degree of relative risk aversion. When $\alpha=1$ a bidder is risk-neutral, the smaller the value of $\alpha$, the more risk-averse is the bidder.

The maximization problem of a bidder with valuation $v$ becomes equivalent to the equation (1.3) except that the payoffs are now scaled by the reverse degree of risk aversion:

$$
\begin{aligned}
& \max _{c} 2\left(\int_{\underline{v}}^{c} \int_{\underline{v}}^{x} \frac{(v-x)^{\alpha}}{\alpha} f(x) f(y) d y d x+\int_{c}^{p} \int_{\underline{v}}^{c} \frac{(v-x)^{\alpha}}{\alpha} f(x) f(y) d y d x+\right. \\
& +\int_{c}^{p} \int_{c}^{x} \frac{(v-p)^{\alpha}}{\alpha} f(x) f(y) d y d x+\int_{p}^{z^{-1}(c)} \int_{\underline{v}}^{x} \frac{(v-p)^{\alpha}}{\alpha} f(x) f(y) d y d x+ \\
& \left.+\int_{z^{-1}(c)}^{\bar{v}} \int_{\underline{v}}^{z(x)} \frac{(v-p)^{\alpha}}{\alpha} f(x) f(y) d y d x+\int_{z^{-1}(c)}^{\bar{v}} \int_{z(x)}^{z^{-1}(c)} \frac{(v-y)^{\alpha}}{\alpha} f(x) f(y) d y d x\right)
\end{aligned}
$$

Solving this maximization problem the same way as in Appendix B results in the following expression ${ }^{6}$ :

$$
z^{\prime}(v)=\frac{f(v)}{f(c)} \frac{\frac{(v-p)^{\alpha}}{\alpha}(F(c)-F(v))+\int_{c}^{v}(v-y) f(y) d y}{F(c) \frac{(c-p)^{\alpha}}{\alpha}+\int_{c}^{p} F(x) d x}
$$

The condition on the negativity of the slope could be reformulated as: $E(v-$ $y \mid y \in[c, v])<\frac{(v-p)^{\alpha}}{\alpha}$ or in other words, the expectation of the payoff received from the third bidders' valuation should be less than the expectation of the payoff received from the posted price conditional on the reverse degree of relative risk-aversion. As $\alpha \downarrow$ the condition becomes more stringent to satisfy and thus the slope increases. Alternatively, $F(c)-F(v)<0$ and $v-p>0$ so a decrease in $\alpha$ makes the numerator

\footnotetext{
${ }^{6}$ Note that just like in $n$-bidders case I am not able to separate valuations so condition is derived in terms of payoffs.
} 
larger. Since $c-p<0$ an increase in $\alpha$ makes the denominator smaller. It means that the overall effect in the increase of risk aversion on bidder's desire to exit the auction earlier is unambiguously positive. Hence, the results of my model confirm the similarities between the incentive structure of auctions with a BIN price and models of simultaneous use of auctions and posted prices when the latter are finite.

\subsubsection{Seller's profits}

It is easy to see that by assuming uniform distribution on $[0,1]$ differential equation (3.10) becomes similar to the original equation where I assumed risk-neutrality, which could be solved for a function linear in $v$. In fact, it does not matter which exact function is chosen to demonstrate the impact of risk aversion on profits as long as it is linear. For simplicity, assume that the function is homomorphic to the one received for the case when buyers were risk neutral:

$$
z(v)=\frac{7}{4} p-\left(\frac{3}{4}\right)^{\alpha} v
$$

Now, using this exit function and the seller's optimization problem from the monopoly case I may simulate seller's profits and an optimal price chosen for a mixed mechanism for different values of $\alpha$. To do this, first substitute a random value of $\alpha$ into the equation (3.10). Then, solve this equation for $v(p)$ when $z(v)=0$ to find an indifferent bidder. Furthermore, solve the latter equation for $p$ when $v=1$ to find the threshold level of the posted price when an indifferent bidder exists. Lastly, substitute all calculated parameters into the seller's problem and check whether the 
Figure 3.3: Simulation of profits and optimal prices for different coefficients of reverse degree of relative risk aversion.
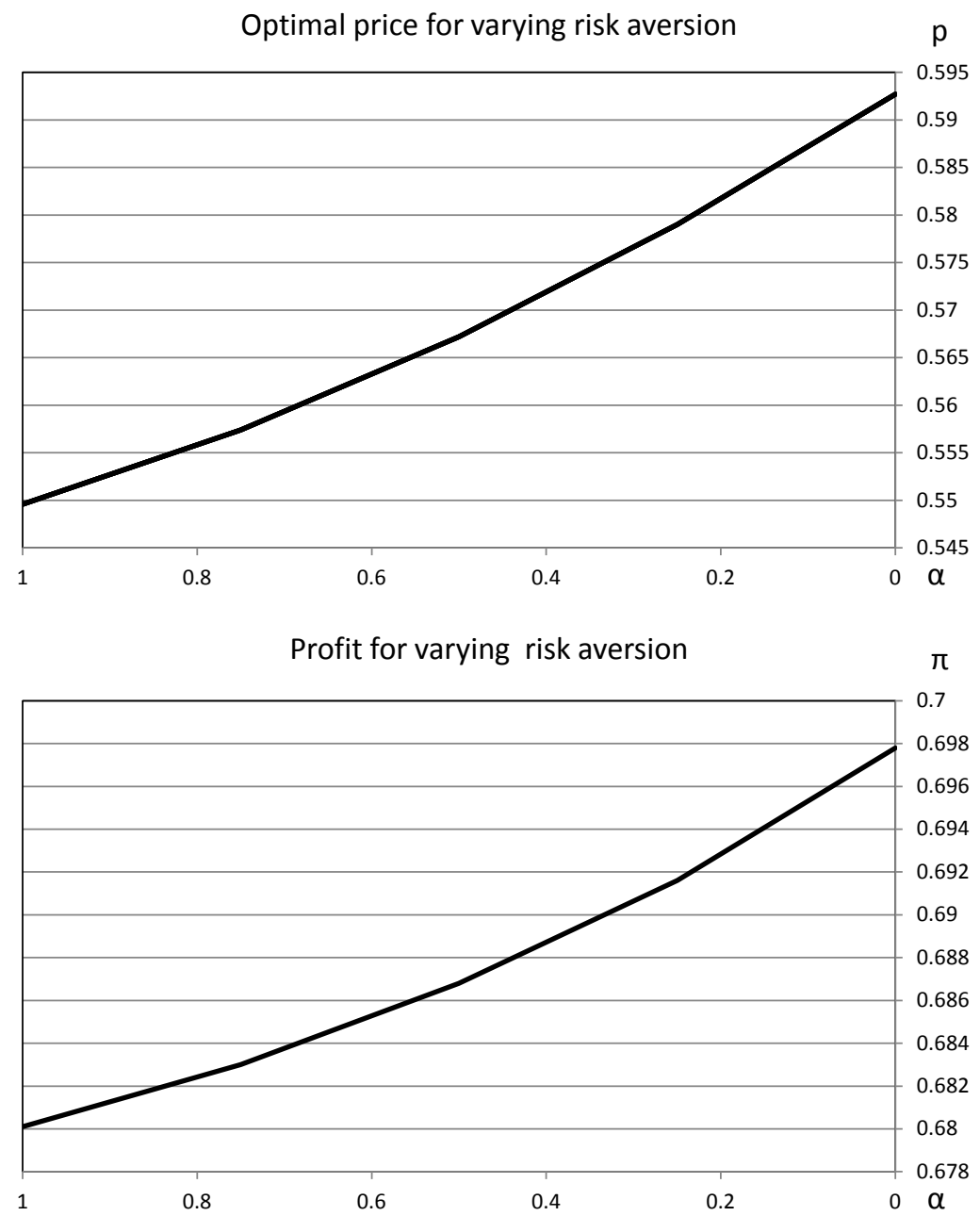
solution satisfies the restriction imposed on $p$.

The results are presented on figure 3.3. As predicted, and similar to the auctions with a BIN price, the optimal price incorporates larger premium with higher degree of risk aversion which eventually results in higher profits. Note also, that when risk aversion is at the maximum $(\alpha=0)$ the profits from using a mixed mechanism are higher than the ones received from using posted prices simultaneously, which was the optimal strategy for risk-neutral buyers.

\subsection{Not observing the number of players}

In the baseline model I have assumed that players are able to observe the number of active bidders in the auction (and in the market per se) two times - at the beginning of the auction, when they choose a bid equal to their exit price, and when the auction price reaches their initial bid. At this point they are able to refine their bid based on the changes in history. Here I analyze the case when bidders are not able to observe their number during the auction, only in the beginning (a common assumption in the literature on auctions with a BIN price). Looking at each integral from the general $3 \times 2$ case notice that only integrals $b(i)$ and $d(i)$ will change.

Recall that in the $\mathrm{b}(\mathrm{i})$ case the second bidder's valuation $x$ was greater than the exit price of the first bidder $c$, but less than the outside posted price, i.e. $c<x<p$ while the third bidder's valuation $y$ was lower than the exit price of the first bidder, i.e. $y<c$. When a bidder was allowed to observe the number of bidders at his exit price he would change his decision to exit the auction since there would only be 2 
bidders left at that time. However, now the bidder with valuation $v$ cannot observe that the bidder with the lowest valuation $y$ has dropped out, so he commits to exiting at price $c$ and receives a payoff of $v-p$ instead of $v-x$. Hence, the whole integral could be rewritten as $\int_{c}^{p} \int_{\underline{v}}^{c}(v-p) 2 f(x) f(y) d y d x$.

In the $\mathrm{d}(\mathrm{i})$ case the second bidder's valuation $x$ was higher than the valuation of the first bidder $z^{-1}(c)<x$, while the third bidder's valuation was below the exit price of the second bidder, i.e. $y<z(x)$. When the second bidder was able to observe the number of bidders at his exit price he would not exit the auction since the bidder with the third valuation would have already dropped out at that point. However, now, he does not observe the number of remaining bidders and exits the auction, which is won by the first bidder. The latter pays the price equal to the exit price of the second bidder, i.e. $z(x)$. Therefore the whole integral could be rewritten as $\int_{z^{-1}(c)}^{\bar{v}} \int_{\underline{v}}^{z(x)}(v-z(x)) 2 f(x) f(y) d y d x$

The maximization problem of the bidder with valuation $v$ could thus be formulated as follows:

$$
\begin{gathered}
\max _{c} 2\left(\int_{\underline{v}}^{c} \int_{\underline{v}}^{x}(v-x) f(x) f(y) d y d x+\int_{c}^{z^{-1}(c)} \int_{\underline{v}}^{x}(v-p) f(x) f(y) d y d x\right. \\
\left.+\int_{z^{-1}(c)}^{\bar{v}} \int_{\underline{v}}^{z(x)}(v-z(x)) f(x) f(y) d y d x+\int_{z^{-1}(c)}^{\bar{v}} \int_{z(x)}^{z^{-1}(c)}(v-y) f(x) f(y) d y d x\right)
\end{gathered}
$$

From Appendix B I already know that $\frac{d}{d c} \int_{\underline{v}}^{c} \int_{\underline{v}}^{x}(v-x) f(x) f(y) d y d x=(v-$ c) $f(c) F(c)$ and $\frac{d}{d c} \int_{z^{-1}(c)}^{\bar{v}} \int_{z(x)}^{z^{-1}(c)}(v-y) f(x) f(y) d y d x=\frac{f\left(z^{-1}(c)\right.}{z^{\prime}\left(z^{-1}(c)\right)}\left(\left(v-z^{-1}(c)\right) \int_{z^{-1}(c)}^{1} f(x) d x-\right.$ $\left.\left.\left(\left(v-z^{-1}(c)\right) F\left(z^{-1}(c)\right)\right)-(v-c) F(c)+\int_{c}^{z^{-1}(c)} F(y) d y\right)\right)$. 
It is easy to show that $\frac{d}{d c} \int_{c}^{z^{-1}(c)} \int_{\underline{v}}^{x}(v-p) f(x) f(y) d y d x=(v-p)\left(\frac{f\left(z^{-1}(c) F\left(z^{-1}(c)\right)\right.}{z^{\prime}\left(z^{-1}(c)\right)}-\right.$ $f(c) F(c))$ and $\frac{d}{d c} \int_{z^{-1}(c)}^{\bar{v}} \int_{\underline{v}}^{z(x)}(v-z(x)) f(x) f(y) d y d x=-\frac{(v-c) f\left(z^{-1}(c) F(c)\right.}{z^{\prime}\left(z^{-1}(c)\right)}$.

After setting $z^{-1}(c)=v$ and some rearrangements the expression could be written as follows: $(v-c) f(c) F(c)-(v-p) f(c) F(c)=\frac{f(v)}{z^{\prime}(v)}((v-c) F(c)-(v-$ $\left.p) F(v)-(v-c) F(c)+\int_{c}^{v} F(y) d y\right)$ or after opening up the brackets and integrating by parts the internal integral: $f(c) F(c)(p-c)=\frac{f(v)}{z^{\prime}(v)}(-v F(v)+p F(v)+v F(v)-$ $\left.c F(c)-\int_{c}^{v} y f(y) d y\right)$.

Thus, $z^{\prime}(v)$ could be expressed as the following fraction:

$$
z^{\prime}(v)=\frac{f(v)\left(p F(v)-c F(c)-\int_{c}^{v} y f(y) d y\right)}{f(c) F(c)(p-c)}
$$

From equation (3.12) it is readily seen that it is not possible to derive an unambiguous condition for the slope to be negative. While the denominator is positive, the numerator may be either positive or negative. As previously, consider the case when buyers' valuations are distributed uniformly on $[0,1]$. Then (3.12) transforms into:

$$
v^{2}-2 p v+z(v)^{2}-2 z(v)(z(v)-p) z^{\prime}(v)=0
$$

The equation above, to the best of my knowledge, does not have a solution. Even solving the part without the explicit $x$-dependence (i.e. $z(v)-2 z(v) z^{\prime}(v)-$ $2 p z(v)=0$ ) would still lead to a product log function (Lambert W-Function ${ }^{7}$ ), i.e.

\footnotetext{
${ }^{7}$ Weisstein, Eric W. "Lambert W-Function." From MathWorld-A Wolfram Web Resource. http://mathworld.wolfram.com/LambertW-Function.html
} 
$z(v)=p W\left(\frac{e^{\frac{v}{2 p}+\frac{c_{1}}{p}}}{p}\right)$, which does not help in finding a closed-form solution.

Another way to approach it is to use the method for homogeneous equations:

$$
\begin{gathered}
2(p-y(x)) y(x)+\frac{x(x-2 p)+y(x)^{2}}{y^{\prime}(x)}=0 \\
2(y-p) y \frac{d y}{d x}=x(x-2 p)+y^{2}
\end{gathered}
$$

Let $u=\frac{y}{x}$ then $y=x u$ and $\frac{d y}{d x}=x \frac{d u}{d x}+u$ :

$$
\begin{gathered}
2(x u-p) x u\left(x \frac{d u}{d x}+u\right)=x(x-2 p)+(x u)^{2} \\
2(x u-p) x^{2} u \frac{d u}{d x}+2(x u-p) x u^{2}=x(x-2 p)+x^{2} u^{2} \\
2(x u-p) x^{2} u \frac{d u}{d x}=x(x-2 p)-(2 x u-x-2 p) x u^{2} \\
\left(x-2 p-(2 x u-x-2 p) u^{2}\right) \frac{d x}{d u}=2(x u-p) x u \\
\left(\left(1-(2 u-1) u^{2}\right) x+2 p\left(u^{2}-1\right)\right) \frac{d x}{d u} \\
2 u^{2} x^{2}-2 p u x
\end{gathered}
$$

Note that the final equation belongs to an Abel equation of the second kind, which could be converted to the first kind with coordinate transformation, but the solutions to this type of equations are cumbersome and do not provide a closed-form solution to be used in the sellers' game. 


\subsection{Conclusion}

In this thesis I have derived the conditions under which buyers' subgame equilibrium exists in a market where there are three buyers competing for two goods offered by an auction and a posted price. I have also fully characterized the equilibrium for the uniform distribution of types. The model explains why it is the case that when the number of objects is less than the number of buyers in a market with posted prices and auctions it is the best response of a bidder with a valuation higher than the posted price to exit the auction before its price matches the outside posted price. Moreover, bidders with higher valuations prefer to exit earlier, and their decision to do so negatively correlates with the level of the posted price of the outside object. In addition, higher number of bidders and higher degree of risk aversion lead to lower exit prices.

With a closed-form solution for bidders' subgame equilibrium I was able to analyze the best response of the seller when he is a monopolist offering two homogeneous objects and deciding how to sell them, and a duopolist competing with another seller. For the monopoly case I found that using posted prices only earns the seller the highest profits followed by a mixed mechanism and auctions only. For the case of the duopoly I showed that using mixed mechanism is the equilibrium. Moreover, unlike models with effectively infinite supply of goods where mixed mechanism is used to price discriminate low- and high-valuation buyers, on the markets of finite goods, when there is competition among sellers, mixed mechanism is the result of their strategic interaction, which hedges against price undercutting. If duopolists were not 
allowed to use auctions, but were forced to compete only in posted prices, their profits would be lower, while consumer surplus - higher. The last argument calls for further investigation into the optimal fee structure of online trading platforms.

The manuscript provides a theoretical framework for interpreting the results of Hammond (2010) and Einav et al. (2016). On top of behavioral factors, such as convenience, there are purely rational reasons to implement a mechanism of posted prices only if a seller is a monopolist. When the number of objects is not significantly less than the number of buyers and the reservation price of the auction is equal to the marginal cost of the seller, selling goods at posted prices is the best response for a seller who is a monopolist in the market. If there are several sellers, and posted prices prevail on the market with finite goods, it may also be a sign of collusion, because using posted prices earns the highest profits only when sellers coordinate their actions. Note that the collusion does not have to be explicit. Online markets are transparent with perfect price observability and inexorability of punishment. It has been well established in the literature on collusion that such market structure is conducive to tacit collusion. Einav et al. (2016) have found that posted prices dominate auctions nowadays. The equilibrium of the duopoly case in my model provides an intuition for this result as well.

The model also makes several empirically testable predictions for the markets of finite goods. The data required to test them would contain information on the mechanisms chosen by sellers in online markets. For example, in monopolized segments, higher posted prices would be the prevailing mechanism, while in new markets a mixed mechanism would be observed. Some of the other predictions could also be 
tested in a lab, such as the bidders' behavior or the choice of sellers' mechanisms under different institutional settings, the implications for the consumer surplus when sellers compete in either mixed mechanism or posted prices only.

Finally, the thesis was a first step into the domain of markets with auctions and finite posted prices. Further research is required in at least two directions. First, introduction of the reserve prices and understanding of the true optimal mechanism. It is possible that some of the results for the monopoly case will change. By not introducing the reserve price I was considering an ex ante efficient market, which is most likely not the case in reality. However, it should not affect the results for the duopoly since even with a zero reserve price mixed mechanism is the equilibrium. Second, generalizing the results for the competition between sellers (including markets with effectively infinite supply of goods). If they hold in a more relaxed environment, it might be interesting to further investigate the optimal fee structure of online two-sided markets. 


\section{Bibliography}

Anwar, S. and M. Zheng (2015). Posted price selling and online auctions. Games and Economic Behavior 90, 81-92.

Bajari, P. and A. Hortacsu (2003). The winner's curse, reserve prices, and endogenous entry: Empirical insights from ebay auctions. RAND Journal of Economics, $329-355$.

Bauner, C. (2015). Mechanism choice and the buy-it-now auction: A structural model of competing buyers and sellers. International Journal of Industrial Organization 38, 19-31.

Burguet, R. and J. Sákovics (1999). Imperfect competition in auction designs. International Economic Review 40(1), 231-247.

Caldentey, R. and G. Vulcano (2007). Online auction and list price revenue management. Management Science 53(5), 795-813.

Chen, J.-R., K.-P. Chen, C.-F. Chou, and C.-I. Huang (2013). A dynamic model of auctions with buy-it-now: Theory and evidence. The Journal of Industrial Economics 61(2), 393-429. 
Chen, K.-P., S.-H. Ho, C.-H. Liu, and C. M. Wang (2016). Optimal listing strategy in online auctions.

Davidson, C. and R. Deneckere (1984). Horizontal mergers and collusive behavior. International Journal of Industrial Organization 2(2), 117-132.

Edgeworth, F. Y. (1897). La teoria pura del monopolio. Giornale degli economisti, $13-31$.

Eeckhout, J. and P. Kircher (2010). Sorting versus screening: Search frictions and competing mechanisms. Journal of Economic Theory 145(4), 1354-1385.

Einav, L., C. Farronato, J. Levin, and N. Sundaresan (2016). Auctions versus posted prices in online markets. Forthcoming Journal of Political Economy.

Etzion, H. and S. Moore (2013). Managing online sales with posted price and open-bid auctions. Decision Support Systems 54(3), 1327-1339.

Etzion, H., E. Pinker, and A. Seidmann (2006). Analyzing the simultaneous use of auctions and posted prices for online selling. Manufacturing \&3 Service Operations Management 8(1), 68-91.

Hafalir, I. and V. Krishna (2008). Asymmetric auctions with resale. American Economic Review 98(1), 87-112.

Hammond, R. G. (2010). Comparing revenue from auctions and posted prices. International Journal of Industrial Organization 28(1), 1-9. 
Hummel, P. (2015). Simultaneous use of auctions and posted prices. European Economic Review 78, 269-284.

Kirkegaard, R. and P. B. Overgaard (2008). Buy-out prices in auctions: seller competition and multi-unit demands. The RAND Journal of Economics 39(3), $770-789$.

Kreps, D. M. and J. A. Scheinkman (1983). Quantity precommitment and bertrand competition yield cournot outcomes. The Bell Journal of Economics, 326-337.

Levitan, R. and M. Shubik (1972). Price duopoly and capacity constraints. International Economic Review, 111-122.

List, J. A. (2004). Neoclassical theory versus prospect theory: Evidence from the marketplace. Econometrica 72(2), 615-625.

Marshall, R. C., M. J. Meurer, J.-F. Richard, and W. Stromquist (1994). Numerical analysis of asymmetric first price auctions. Games and Economic Behavior 7(2), $193-220$.

Mathews, T. and B. Katzman (2006). The role of varying risk attitudes in an auction with a buyout option. Economic Theory 27(3), 597-613.

McAfee, R. P. (1993). Mechanism design by competing sellers. Econometrica: Journal of the econometric society, 1281-1312.

Ockenfels, A. and A. E. Roth (2006). Late and multiple bidding in second price 
internet auctions: Theory and evidence concerning different rules for ending an auction. Games and Economic behavior 55(2), 297-320.

Osborne, M. J. and C. Pitchik (1986). Price competition in a capacity-constrained duopoly. Journal of Economic Theory 38(2), 238-260.

Peters, M. and S. Severinov (1997). Competition among sellers who offer auctions instead of prices. Journal of Economic Theory 75(1), 141-179.

Peters, M. and S. Severinov (2006). Internet auctions with many traders. Journal of Economic Theory 130(1), 220-245.

Reynolds, S. S. and J. Wooders (2009). Auctions with a buy price. Economic Theory 38(1), 9-39.

Sun, D. (2008). Dual mechanism for an online retailer. European Journal of Operational Research 187(3), 903-921.

Van Essen, M. and J. Wooders (2016). Dissolving a partnership dynamically. Journal of Economic Theory 166, 212-241.

Vickrey, W. (1961). Counterspeculation, auctions, and competitive sealed tenders. The Journal of finance 16(1), 8-37.

Virág, G. (2010). Competing auctions: finite markets and convergence. Theoretical Economics 5(2), 241-274.

Vives, X. (1986). Rationing rules and bertrand-edgeworth equilibria in large markets. Economics Letters 21(2), 113-116. 
Appendices 


\section{Appendix A}

\section{Expected payoff of the bidder with}

\section{valuation $v$}

There are several possibilities where the valuations of rivaling bidders could be realized:

a) Both other bidders' valuations are below the exit price of the first bidder: $x, y<c$. In this situation bidding up to their valuations is the best response. Both bidders drop out when the auction price reaches their valuations. Bidder one wins the auction and pays the second highest bid. His expected revenue is equal to:

$$
\int_{\underline{v}}^{c} \int_{\underline{v}}^{x}(v-x) 2 f(x) f(y) d y d x^{1}
$$

\footnotetext{
${ }^{1}$ Note that this and the following integrals could be rewritten in the following way: $2 \int_{\underline{v}}^{c}\left(\int_{\underline{v}}^{x}(v-\right.$ $x) f(y) d y) f(x) d x$, where the inner integral shows the expected payoff with variation of the third bidder's valuation $y$ on the interval from $\underline{v}$ to $c$ and the external one - payoff from the variation of the second bidder's valuation $x$ on the same interval taking into account corresponding joint density $2 f(x) f(y)$.
} 
b) (i) Second bidder's valuation is greater than the exit price of the first bidder $x>c$, but less than the outside posted price $c<x<p$, while the third bidder's valuation is lower than the exit price of the first bidder $y<c$. In this scenario, when the auction price reaches the level of the third bidder's valuation $q=y$, he drops out. At $q=c$ the first bidder observes that one of the bidders has left the auction, while the good is still available at the posted price. He no longer exits the auction and follows the equilibrium strategy of the $2 \times 2$ case. Since the second bidder's valuation is below the posted price, he stays until the auction prices reaches his valuation $q=x$ and then drops out. The first bidder pays the second highest bid and receives the following expected payoff:

$$
\int_{c}^{p} \int_{\underline{v}}^{c}(v-x) 2 f(x) f(y) d y d x
$$

(ii) Second bidder's valuation is greater than the exit price of the first bidder, but less than the outside posted price $(c<x<p)$, while the third bidder's valuation is also greater than the exit price of the first bidder, but lower than the valuation of the second bidder $(c<y<x)$. In this case, when the auction price reaches the exit price of the first bidder, he leaves the auction and buys the good at the posted price:

$$
\int_{c}^{p} \int_{c}^{x}(v-p) 2 f(x) f(y) d y d x
$$

c) (i) Second bidder's valuation is above the posted price, but lower than the "reported valuation" ${ }^{2}$ of the first bidder $\left(p<x<z^{-1}(c)\right)$, while the third bidder's

\footnotetext{
${ }^{2}$ In essence, this is a direct mechanism for which buyers submit their valuations, and it provides
} 
valuation is lower than the exit price of the first bidder $(y<c)$. This situation is similar to the one described in $\mathrm{b}(\mathrm{i})$ : the auction continues until the auction price reaches the valuation of the third bidder: he exits the auction, and the remaining bidders play according to the $2 \times 2$ case. Bidding continues until the auction price matches the outside posted price. At price $q=p$ one bidder leaves the auction and buys the good at the posted price, while the other one wins the auction and pays the same amount:

$$
\int_{p}^{z^{-1}(c)} \int_{\underline{v}}^{c}(v-p) 2 f(x) f(y) d y d x
$$

(ii) The second bidder's valuation is above the posted price level, but lower than the "reported valuation" of the first bidder $\left(p<x<z^{-1}(c)\right)$, while the third bidder's valuation is between the exit price for the first bidder and the posted price $(c<y<p)$. In this case, when the auction price reaches the level of the exit price for the first bidder $(q=c)$, he leaves the auction and buys the good at a posted price:

$$
\int_{p}^{z^{-1}(c)} \int_{c}^{p}(v-p) 2 f(x) f(y) d y d x
$$

(iii) The second bidder's valuation is still above the posted price level, but lower than the "reported valuation" of the first bidder $\left(p<x<z^{-1}(c)\right)$, while the third bidder's valuation is between the posted price level and the second bidder's valuation $(p<y<x)$. Nothing changes from c(ii) — first bidder is still the first to leave the them with exit prices. Of course, in equilibrium, the "reported valuation" will be the true value maximizing the payoff of the buyer with valuation $v$. 
auction and buy the good at the posted price:

$$
\int_{p}^{z^{-1}(c)} \int_{p}^{x}(v-p) 2 f(x) f(y) d y d x
$$

Note that the last three expected payoffs can be summed up to:

$$
\int_{p}^{z^{-1}(c)} \int_{\underline{v}}^{x}(v-p) 2 f(x) f(y) d y d x
$$

d) (i) The second bidder's valuation is higher than the "reported valuation" of the first bidder $\left(z^{-1}(c)<x\right)$, while the third bidder's valuation is below the exit price of the second bidder, which is symmetrically mapped onto the valuation axis via the exit function $z(x)$. In this case the third bidder leaves the auction, and the game returns to the $2 \times 2$ case. The auction continues until its price matches the outside posted price. At $q=p$ one bidder leaves the auction and buys the good at the posted price, while the other one wins the auction and pays the same amount:

$$
\int_{z^{-1}(c)}^{\bar{v}} \int_{\underline{v}}^{z(x)}(v-p) 2 f(x) f(y) d y d x
$$

(ii) The second bidder's valuation is above the "reported valuation" of the first bidder $\left(z^{-1}(c)<x\right)$, while the third bidder's valuation is between the exit price of the second bidder and the exit price of the first bidder $(z(x)<y<c)$. In this scenario, the second bidder leaves the auction and buys the good at the posted price. The remaining bidders are back to the $2 \times 2$ case. When $q$ reaches the valuation of the third bidder $y<p$ the first bidder wins the auction and receives a payoff of $v-y$. 
Since the good outside of the auction is no longer available it is readily seen that the same outcome will also be observed on $c<y<z^{-1}(c)$. Thus, the total expected payoff of the first bidder for this case is:

$$
\int_{z^{-1}(c)}^{\bar{v}} \int_{z(x)}^{z^{-1}(c)}(v-y) 2 f(x) f(y) d y d x
$$

(iii) The second bidder's valuation is above the "reported valuation" of the first bidder $\left(z^{-1}(c)<x\right)$ and the third bidder's valuation is also above the first bidder's valuation, but below the second bidder's valuation $\left(z^{-1}(c)<y<x\right)$. It is easy to see that given the assumption of symmetry the first bidder will not be able to get the good from either the auction or the posted price and will receive a payoff of 0 . 


\section{Appendix B}

\section{Proof of Lemma 3}

To begin note that both parts of the equation can be divided by 2 :

$$
\begin{gathered}
\frac{d}{d c} \int_{\underline{v}}^{c} \int_{\underline{v}}^{x}(v-x) f(x) f(y) d y d x+\frac{d}{d c} \int_{c}^{p} \int_{\underline{v}}^{c}(v-x) f(x) f(y) d y d x+ \\
\frac{d}{d c} \int_{c}^{p} \int_{c}^{x}(v-p) f(x) f(y) d y d x+\frac{d}{d c} \int_{p}^{z^{-1}(c)} \int_{\underline{v}}^{x}(v-p) f(x) f(y) d y d x+ \\
\frac{d}{d c} \int_{z^{-1}(c)}^{\bar{v}} \int_{\underline{v}}^{z(x)}(v-p) f(x) f(y) d y d x+\frac{d}{d c} \int_{z^{-1}(c)}^{\bar{v}} \int_{z(x)}^{z^{-1}(c)}(v-y) f(x) f(y) d y d x=0
\end{gathered}
$$

Applying fundamental theorem of calculus to each of the integrals and in some instances integrating by parts I get ${ }^{1}$ :

\footnotetext{
${ }^{1}$ Note that $F(\underline{v})=0$ and $F(\bar{v})=1$ and $\frac{d}{d c} z^{-1}(c)=\frac{1}{z^{\prime}\left(z^{-1}(c)\right)}$.
} 


$$
\begin{gathered}
\frac{d}{d c} \int_{\underline{v}}^{c} \int_{\underline{v}}^{x}(v-x) f(x) f(y) d y d x=\frac{d}{d c} \int_{\underline{v}}^{c}(v-x) f(x) \int_{\underline{v}}^{x} f(y) d y d x= \\
\frac{d}{d c} \int_{\underline{v}}^{c}(v-x) f(x) F(x) d x=(v-c) f(c) F(c)
\end{gathered}
$$

$$
\begin{gathered}
\frac{d}{d c} \int_{c}^{p} \int_{\underline{v}}^{c}(v-x) f(x) f(y) d y d x=\frac{d}{d c} F(c) \int_{c}^{p}(v-x) f(x) d x= \\
f(c) \int_{c}^{p}(v-x) f(x) d x-F(c) f(c)(v-c)= \\
f(c)\left((v-p) F(p)-(v-c) F(c)+\int_{c}^{p} F(x) d x-F(c)(v-c)\right)= \\
f(c)\left((v-p) F(p)-2(v-c) F(c)+\int_{c}^{p} F(x) d x\right)
\end{gathered}
$$

$$
\begin{gathered}
\frac{d}{d c} \int_{c}^{p} \int_{c}^{x}(v-p) f(x) f(y) d y d x=(v-p) \frac{d}{d c} \int_{c}^{p} f(x)(F(x)-F(c)) d x= \\
(v-p) \frac{d}{d c}\left(\int_{c}^{p} f(x) F(x) d x-F(c) \int_{c}^{p} f(x) d x\right)= \\
(v-p)\left(-f(c) F(c)-\frac{d}{d c} F(c)(F(p)-F(c))\right)= \\
(v-p)(-f(c) F(c)-f(c)(F(p)-F(c))+f(c) F(c))= \\
-(v-p) f(c)(F(p-F(c))
\end{gathered}
$$

$$
\begin{gathered}
\frac{d}{d c} \int_{p}^{z^{-1}(c)} \int_{\underline{v}}^{x}(v-p) f(x) f(y) d y d x=\frac{d}{d c} \int_{p}^{z^{-1}(c)}(v-p) f(x) F(x) d x= \\
\frac{(v-p) f\left(z^{-1}(c)\right) F\left(z^{-1}(c)\right)}{z^{\prime}\left(z^{-1}(c)\right)}
\end{gathered}
$$




$$
\begin{gathered}
\frac{d}{d c} \int_{z^{-1}(c)}^{\bar{v}} \int_{\underline{v}}^{z(x)}(v-p) f(x) f(y) d y d x=\frac{d}{d c} \int_{z^{-1}(c)}^{\bar{v}}(v-p) f(x) F(z(x)) d x= \\
-\frac{(v-p) f\left(z^{-1}(c)\right) F(c)}{z^{\prime}\left(z^{-1}(c)\right)} \\
\frac{d}{d c} \int_{z^{-1}(c)}^{\bar{v}} \int_{z(x)}^{z^{-1}(c)}(v-y) f(x) f(y) d y d x=-\frac{f\left(z^{-1}(c)\right.}{z^{\prime}\left(z^{-1}(c)\right)} \int_{c}^{z^{-1}(c)}(v-y) f(y) d y+ \\
\frac{\left(v-z^{-1}(c)\right) f\left(z^{-1}(c)\right)}{z^{\prime}\left(z^{-1}(c)\right)} \int_{z^{-1}(c)}^{1} f(x) d x=\frac{f\left(z^{-1}(c)\right.}{z^{\prime}\left(z^{-1}(c)\right)}\left(\left(v-z^{-1}(c)\right) \int_{z^{-1}(c)}^{1} f(x) d x-\right. \\
\left.\left.\left(\left(v-z^{-1}(c)\right) F\left(z^{-1}(c)\right)\right)-(v-c) F(c)+\int_{c}^{z^{-1}(c)} F(y) d y\right)\right)
\end{gathered}
$$

Substituting each integral back into the original equation produces:

$$
\begin{gathered}
(v-c) f(c) F(c)+(v-p) f(c) F(p)-2(v-c) f(c) F(c)+f(c) \int_{c}^{p} F(x) d x \\
-(v-p) f(c)(F(p)-F(c))+\frac{(v-p) f\left(z^{-1}(c)\right) F\left(z^{-1}(c)\right)}{z^{\prime}\left(z^{-1}(c)\right)} \\
-\frac{(v-p) f\left(z^{-1}(c)\right) F(c)}{z^{\prime}\left(z^{-1}(c)\right)}+\frac{f\left(z^{-1}(c)\right)}{z^{\prime}\left(z^{-1}(c)\right)}\left(( v - z ^ { - 1 } ( c ) ) \left(1-F\left(z^{-1}(c)\right)\right.\right. \\
\left.-\left(\left(v-z^{-1}(c)\right) F\left(z^{-1}(c)\right)-(v-c) F(c)+\int_{c}^{z^{-1}(c)} F(y) d y\right)\right)=0
\end{gathered}
$$

In the equilibrium $z^{-1}(c)=v$ and $c=z(v)$. Therefore, some elements with $v-z^{-1}(c)$ turn into 0 , and after some rearrangements the equation becomes: 


$$
\begin{gathered}
f(c)\left(F(c)(c-p)+\int_{c}^{p} F(x) d x\right)= \\
\frac{f(v)\left((v-p)(F(c)-F(v))-(v-c) F(c)+\int_{c}^{v} F(y) d y\right)}{z^{\prime}(v)}
\end{gathered}
$$

Expressing the derivative and further simplifying the numerator produces:

$$
z^{\prime}(v)=\frac{f(v)}{f(c)} \frac{F(c)(c-p)-F(v)(v-p)+\int_{c}^{v} F(y) d y}{F(c)(c-p)+\int_{c}^{p} F(x) d x}
$$

Finally, integrating by parts $\int_{c}^{v} F(y) \times 1 d y$ I can rewrite the numerator as $F(c)(c-p)-F(v)(v-p)+F(v) v-F(c) c-\int_{c}^{v} y f(y) d y$ or equivalently $p(F(v)-$ $F(c))-\int_{c}^{v} y f(y) d y$

Now I will prove the following properties of this equation:

a) The function $z(v)$ is bounded upwardly by $p$.

If $c=p$ then it is easy to see that the denominator of equation (B.9) becomes zero and the derivative non-existent. If $c>p$ then $\int_{c}^{p} F(x) d x$ becomes undefined. Therefore, the derivative exists only when $c<p$ and hence the function itself. When $v=p$ it must be that $c=p$ and consequently the derivative is not defined for all $v \leq p$

b) The slope of the function $z(v)$ is negative if and only if $p<E(y) \mid y \in[c, v]$, in other words, a bidder exits the auction before its price matches the posted price outside if and only if the expectation of the lowest bidder's valuation between the value of the exit function and the valuation of the former bidder is higher than the posted price.

The denominator of (B.9) is positive, because $F(c)(c-p)+\int_{c}^{p} F(x) d x>$ $F(c)(c-p)+\int_{c}^{p} F(c) d x=0$. Hence, for the whole fraction to be negative the 
numerator should also be negative which happens only when $p(F(v)-F(c))<$ $\int_{c}^{v} y f(y) d y$ or $p<\frac{\int_{c}^{v} y f(y) d y}{F(v)-F(c)}$ where the latter term is the expected value of the third buyer on the interval $[c, v]$.

c) The slope of the function $z(v)$ with respect to $p$ is negative.

Considering only negative $z^{\prime}(v)$ notice that $F(v)-F(c)$ is positive. Hence, the numerator decreases as $p$ rises and increases if $p$ falls. From $F(c)(c-p)+\int_{c}^{p} F(x) d x>$ $F(c)(c-p)+\int_{c}^{p} F(c) d x=0$ it is also seen that the denominator increases as $p$ rises and vice versa. 


\section{Appendix C}

\section{Expected seller's revenue from selling}

\section{both objects as a monopolist}

a) Every bidder's valuation is below the posted price, i.e. $v, x, y<p$. From Lemma 1 I know that the seller's expected revenue from the auction will be equal to the second highest valuation. Since bidders cannot afford the good at the posted price his profit from the posted price is zero:

$$
6 \int_{0}^{p} \int_{0}^{v} \int_{0}^{x}(x+0) d y d x d v
$$

b) First bidder's valuation is higher than the posted price, but below the value

of the indifferent bidder i.e. $p<v<\frac{7}{3} p$ while the valuations of the other bidders are below the exit price of the first bidder $(y<x<c)$. In this case both bidders drop out before the auction price reaches the exit price of the first bidder and he wins the auction paying the second highest valuation. 


$$
6 \int_{p}^{\frac{7}{3} p} \int_{0}^{c} \int_{0}^{x}(x+0) d y d x d v
$$

c) (i) First bidder's valuation is higher than the posted price, but below the value of the indifferent bidder i.e. $p<v<\frac{7}{3} p$; second bidder's valuation is higher than the exit price of the first bidder, but lower than the posted price $(c<x<p)$; third bidder's valuation is lower than the exit price $(y<c)$. In this case the bidder with the lowest valuation drops out before the auction price reaches the exit price of the first bidder, and the first bidder continues bidding in the auction. He wins it and pays the second highest valuation:

$$
6 \int_{p}^{\frac{7}{3} p} \int_{c}^{p} \int_{0}^{c}(x+0) d y d x d v
$$

(ii) First bidder's valuation is higher than the posted price, but below the valuation of the indifferent bidder i.e. $p<v<\frac{7}{3} p$; second bidder's valuation is higher than the exit price of the first bidder, but lower than the posted price $(c<x<p)$; third bidder's valuation is higher than the exit price of the first bidder, but lower than the valuation of the second bidder $(c<y<x)$. In this case, the first bidder exits the auction and buys the good at the posted price, while the auction is won by the second bidder who pays the third bidder's valuation:

$$
6 \int_{p}^{\frac{7}{3} p} \int_{c}^{p} \int_{c}^{x}(y+p) d y d x d v
$$

d) (i) First bidder's valuation is higher than the posted price, but below the 
value of the indifferent bidder i.e. $p<v<\frac{7}{3} p$; second bidder's valuation is higher than the posted price, but lower than the valuation of the first bidder $(p<x<v)$; third bidder's valuation is below the exit price of the first bidder $(y<c)$. In this case the bidder with the lowest valuation drops out before the auction price reaches the exit price of the first bidder, and the latter continues to bid in the auction according to the $2 \times 2$ case. The auction price rises until it matches the posted price outside. One of the remaining bidders wins the auction, the other one - buys the good at the posted price, they both pay the price $p$ :

$$
6 \int_{p}^{\frac{7}{3} p} \int_{p}^{v} \int_{0}^{c}(p+p) d y d x d v
$$

(ii) First bidder's valuation is higher than the posted price, but below the value of the indifferent bidder i.e. $p<v<\frac{7}{3} p$; second bidder's valuation is higher than the posted price, but lower than the valuation of the first bidder $(p<x<v)$; third bidder's valuation is higher than the exit price of the first bidder, but lower than the posted price outside $(c<y<p)$. In this case, the first bidder exits the auction at his exit price and buys the good outside. The auction continues with the other bidders bidding up until the valuation of the third bidder:

$$
6 \int_{p}^{\frac{7}{3} p} \int_{p}^{v} \int_{c}^{p}(y+p) d y d x d v
$$

(iii) First bidder's valuation is higher than the posted price, but below the value of the indifferent bidder i.e. $p<v<\frac{7}{3} p$; second bidder's valuation is higher than the posted price, but lower than the valuation of the first bidder $(p<x<v)$; 
third bidder's valuation is higher than the posted price, but lower than the valuation of the second bidder $(p<y<x)$. In this case the first bidder exits the auction at his exit price and buys the good outside. The auction continues with the other bidders bidding up until the valuation of the third bidder:

$$
6 \int_{p}^{\frac{7}{3} p} \int_{p}^{v} \int_{p}^{x}(y+p) d y d x d v
$$

f) First bidder's valuation is higher than the valuation of the indifferent bidder, i.e. $\frac{7}{3} p<v<1$, while the second and third bidder's valuations are distributed accordingly on $[0, v]$ and $[0, x]$. In this case, the first bidder does not participate in the auction and buys the good at the posted price right away. The auction is carried out between the other two bidders who bid their valuations. The auction ends at the valuation of the third bidder.

$$
6 \int_{\frac{7}{3} p}^{1} \int_{0}^{v} \int_{0}^{x}(y+p) d y d x d v
$$




\section{Appendix D}

\section{Proof of Lemma 8}

Substituting the joint density of $(n-1)$ and density of the order statistics of the maximum of $(n-2)$ I get the following optimization problem for a bidder:

$$
\begin{gathered}
\quad \frac{d}{d c} \int_{\underline{v}}^{c} \int_{\underline{v}}^{x}(v-x)(n-1) f(x)(n-2) F(y)^{n-3} f(y) d y d x \\
+\frac{d}{d c} \int_{c}^{p} \int_{\underline{v}}^{c}(v-x)(n-1) f(x)(n-2) F(y)^{n-3} f(y) d y d x \\
+\frac{d}{d c} \int_{c}^{p} \int_{c}^{x}(v-p)(n-1) f(x)(n-2) F(y)^{n-3} f(y) d y d x \\
+\frac{d}{d c} \int_{p}^{z^{-1}(c)} \int_{\underline{v}}^{x}(v-p)(n-1) f(x)(n-2) F(y)^{n-3} f(y) d y d x \\
+\frac{d}{d c} \int_{z^{-1}(c)}^{\bar{v}} \int_{\underline{v}}^{z(x)}(v-p)(n-1) f(x)(n-2) F(y)^{n-3} f(y) d y d x \\
+\frac{d}{d c} \int_{z^{-1}(c)}^{\bar{v}} \int_{z(x)}^{z^{-1}(c)}(v-y)(n-1) f(x)(n-2) F(y)^{n-3} f(y) d y d x=0
\end{gathered}
$$

Notice that $\int F(y)^{n-3} f(y) d y=\int F(y)^{n-3} d F(y)=\frac{F(y)^{n-2}}{n-2}$. Solving each inte- 
gral separately produces:

$$
\begin{aligned}
& \frac{d}{d c} \int_{\underline{v}}^{c}(n-1)(v-x) f(x) F(x)^{n-2} d x=(n-1)(v-c) f(c) F(c)^{n-2} \\
& \frac{d}{d c} F(c)^{n-2}(n-1) \int_{c}^{p}(v-x) f(x) d x= \\
& (n-1)\left((n-2) F(c)^{n-3} f(c) \int_{c}^{p}(v-x) f(x) d x-(v-c) f(c) F(c)^{n-2}\right)= \\
& (n-1) f(c) F(c)^{n-3}\left((n-2)\left((v-p) F(p)-(v-c) F(c)+\int_{c}^{p} F(x) d x\right)-(v-c) F(c)\right) \\
& \frac{d}{d c} \int_{c}^{p}(n-1)(v-p) f(x)\left(F(x)^{n-2}-F(c)^{n-2}\right) d x= \\
& (n-1)(v-p) \frac{d}{d c}\left(\int_{c}^{p} f(x) F(x)^{n-2} d x-F(c)^{n-2} \int_{c}^{p} f(x) d x\right)= \\
& (n-1)(v-p)\left(-f(c) F(c)^{n-2}-\left((n-2) F(c)^{n-3} f(c)(F(p)-F(c))-F(c)^{n-2} f(c)\right)\right)= \\
& -(n-1)(n-2)(v-p) F(c)^{n-3} f(c)(F(p)-F(c)) \\
& \frac{d}{d c} \int_{p}^{z^{-1}(c)}(n-1)(v-p) f(x) F(x)^{n-2} d x=\frac{(n-1)(v-p) F\left(z^{-1}(c)\right)^{n-2} f\left(z^{-1}(c)\right)}{z^{\prime}\left(z^{-1}(c)\right)}
\end{aligned}
$$




$$
\begin{gathered}
\frac{d}{d c} \int_{z^{-1}(c)}^{\bar{v}}(n-1)(v-p) f(x) F(z(x))^{n-2} d x=\frac{-(n-1)(v-p) F(c)^{n-2} f\left(z^{-1}(c)\right)}{z^{\prime}\left(z^{-1}(c)\right)} \\
-\frac{f\left(z^{-1}(c)\right)(n-1)(n-2)}{z^{\prime}\left(z^{-1}(c)\right)}\left(\int_{c}^{z^{-1}(c)}(v-y) F(y)^{n-3} f(y) d y+\right. \\
\left.\left(v-z^{-1}(c)\right) f\left(z^{-1}(c)\right) F\left(z^{-1}(c)\right)^{n-3} \int_{z^{-1}(c)}^{\bar{v}} f(x) d x\right)
\end{gathered}
$$

Substituting back and setting $z^{-1}(c)=v$ I receive the following equation:

$$
\begin{gathered}
f(c) F(c)^{n-3}(n-2)\left((v-p) F(p)-(v-c) F(c)+\int_{c}^{p} F(x) d x\right)- \\
(v-p)(n-2) f(c) F(c)^{n-3}(F(p)-F(c))=\frac{f(v)}{z^{\prime}(v)}\left((v-p) F(c)^{n-2}-\right. \\
\left.(v-p) F(v)^{n-2}+(n-2) \int_{c}^{z^{-1}(c)}(v-y) F(y)^{n-3} f(y) d y\right)
\end{gathered}
$$

Opening the brackets and simplifying the left-hand side of the equation produces:

$$
(n-2) f(c) F(c)^{n-3}\left((c-p) F(c)+\int_{c}^{p} F(x) d x\right)
$$

And the right-hand side:

$$
\frac{f(v)}{z^{\prime}(v)}\left((v-p)\left(F(c)^{n-2}-F(v)^{n-2}\right)+(n-2) \int_{c}^{z^{-1}(c)}(v-y) F(y)^{n-3} f(y) d y\right)
$$

Expressing the derivative from the above LHS and RHS gives the result: 


$$
z^{\prime}(v)=\theta \frac{\left.(v-p)\left(F(c)^{n-2}-F(v)^{n-2}\right)+(n-2) \int_{c}^{v}(v-y) F(y)^{n-3} f(y) d y\right)}{F(c)(c-p)+\int_{c}^{p} F(x) d x}
$$

where $\theta=\frac{f(v)}{(n-2) f(c) F(c)^{n-3}}>0$. Now, I will prove stated properties:

a) The function $z(v)$ exists only if $c<p$.

If $c=p$ then it is easy to see that the denominator of equation (D.8) becomes 0 and the derivative non-existent. If $c>p$ then $\int_{c}^{p} F(x) d x$ becomes undefined. Therefore, the derivative exists only when $c<p$ and hence the function itself.

b) The function $z(v)$ is negative only if $v-p>E(v-y)$ on $[c, v]$., where $y$ is the expected value of the maximum of the order statistics of $n-2$

The denominator of (D.8) is positive, because $F(c)(c-p)+\int_{c}^{p} F(x) d x>$ $F(c)(c-p)+\int_{c}^{p} F(c) d x=0$. Hence, for the whole fraction to be negative the numerator should also be negative. Notice that $F(c)^{n-2}-F(v)^{n-2}$ is negative while $v-p$ - positive. $(n-2) \int_{c}^{v}(v-y) F(y)^{n-3} f(y) d y$ is positive. Therefore, rewrite the numerator as follows: $\left.(n-2) \int_{c}^{v}(v-y) F(y)^{n-3} f(y) d y\right)-(v-p)\left(F(v)^{n-2}-F(c)^{n-2}\right)$. For the numerator to be positive it has to be that: $v-p>\frac{(n-2) \int_{c}^{v}(v-y) F(y)^{n-3} f(y) d y}{\left(F(v)^{n-2}-F(c)^{n-2}\right)}$. But $(n-2) F(y)^{n-3} f(y)$ is the order statistics of the maximum of $n-2$. Therefore the fraction above is the expected value of the maximum of the order statistics of $n-2$ on the interval $[c, v]$.

c) The slope of the function $z(v)$ increases when the posted price decreases and vice versa.

Considering only negative $z^{\prime}(v)$ it is readily seen that the numerator decreases 
as $p$ rises and increases if $p$ falls. From $F(c)(c-p)+\int_{c}^{p} F(x) d x>F(c)(c-p)+$ $\int_{c}^{p} F(c) d x=0$ it is also seen that the denominator increases as $p$ rises and vice versa.

d) The slope of the function $z(v)$ increases when the number of bidders increases and vice versa.

The whole fraction rises when $n$ increases, but $(n-2) f(c) F(c)^{n-3}<(n-$ 2) $\int_{c}^{v}(v-y) F(y)^{n-3} f(y) d y$ and so the numerator increases faster. 


\section{Appendix E}

\section{Proof of Lemma 9}

To find an analytical approximation to the equation (3.3) I will apply perturbation analysis, which has already been used in auction theory ${ }^{1}$.

The first step is to select a point around which to perturb the equation. In this particular case such point is a point of singularity $(p, p)$, which is derived from the model $^{2}$. Adjust the equation to the center of coordinates by subtracting $p$ from both variables, i.e. define $X=v-p$ and $Y=z-p$. Dropping the argument to simplify the notation results into the following equation:

$$
\frac{1}{2}(p+Y)^{n-3} Y^{2} Y^{\prime}=\frac{(p+X)^{n-2}-(p+Y)^{n-2}}{n-2} p-\frac{(p+X)^{n-1}-(p+Y)^{n-1}}{n-1}
$$

Now, the function is not defined around $(0,0)$. Moreover $\lim _{X \rightarrow 0^{+}} Y=0$ with $X \gg X^{2} \gg X^{3}$. The next step is to rescale the variables by introducing a small

\footnotetext{
${ }^{1}$ See Fibich, Gadi, and Arieh Gavious. "Asymmetric first-price auctions - a perturbation approach." Mathematics of Operations Research 28, no. 4 (2003): 836-852.

${ }^{2}$ Note that it does not have to be a point of singularity for the perturbation purposes.
} 
parameter $\epsilon$, which will help to keep track of their order. $\epsilon^{0}$ indicates leading order, $\epsilon^{1}$ - first perturbed term, $\epsilon^{2}$ - second perturbed term etc... Setting $X=\epsilon x$ and $Y=\epsilon y$ :

$\frac{1}{2}(p+\epsilon y)^{n-3}(\epsilon y)^{2}\left(y^{\prime}+\epsilon y^{\prime}\right)=\frac{(p+\epsilon x)^{n-2}-(p+\epsilon y)^{n-2}}{n-2} p-\frac{(p+\epsilon x)^{n-1}-(p+\epsilon y)^{n-1}}{n-1}$

Next, apply binomial expansion to expand the polynomials according to the standard formula:

$$
(a+b)^{n}=\frac{a^{n}}{0 !}+\frac{n a^{n-1} b}{1 !}+\frac{n(n-1) a^{n-2} b^{2}}{2 !}+\frac{n(n-1)(n-2) a^{n-3} b^{3}}{3 !}+\ldots
$$

To derive an equation with one perturbed term I need to drop anything with $\epsilon^{4}$ and higher since the left part of the equation (E.2) already contains a term $(\epsilon y)^{2}$, which will cancel out with corresponding powers of $\epsilon$ in the right-hand side, leaving only $\epsilon^{0}$ and $\epsilon^{1}$. Applying the expansion to each of the right-hand side brackets (also multiplying by $p$ for the first fraction) I get:

$$
\begin{aligned}
(p+\epsilon x)^{n-2} p= & p^{n-1}+\epsilon(n-2) p^{n-2} x+\frac{1}{2} \epsilon^{2}(n-3)(n-2) p^{n-3} x^{2}+ \\
& +\frac{1}{6} \epsilon^{3}(n-4)(n-3)(n-2) p^{n-4} x^{3}
\end{aligned}
$$




$$
\begin{aligned}
(p+\epsilon y)^{n-2} p= & p^{n-1}+\epsilon(n-2) p^{n-2} y+\frac{1}{2} \epsilon^{2}(n-3)(n-2) p^{n-3} y^{2}+ \\
& \frac{1}{6} \epsilon^{3}(n-4)(n-3)(n-2) p^{n-4} y^{3} \\
(p+\epsilon x)^{n-1}= & p^{n-1}+\epsilon(n-1) p^{n-2} x+\frac{1}{2} \epsilon^{2}(n-2)(n-1) p^{n-3} x^{2}+ \\
& +\frac{1}{6} \epsilon^{3}(n-3)(n-2)(n-1) p^{n-4} x^{3} \\
& \\
& +\frac{1}{6} \epsilon^{3}(n-3)(n-2)(n-1) p^{n-4} y^{3}
\end{aligned}
$$

Substituting the polynomials into the right-hand side of the equation (E.2) many terms cancel out and I am left with the following expression: 


$$
\begin{gathered}
\frac{1}{n-2}\left(p^{n-1}+\epsilon(n-2) p^{n-2} x+\frac{1}{2} \epsilon^{2}(n-3)(n-2) p^{n-3} x^{2}+\right. \\
+\frac{1}{6} \epsilon^{3}(n-4)(n-3)(n-2) p^{n-4} x^{3}-p^{n-1}-\epsilon(n-2) p^{n-2} y- \\
\left.\frac{1}{2} \epsilon^{2}(n-3)(n-2) p^{n-3} y^{2}-\frac{1}{6} \epsilon^{3}(n-4)(n-3)(n-2) p^{n-4} y^{3}\right)- \\
-\frac{1}{n-1}\left(p^{n-1}+\epsilon(n-1) p^{n-2} x+\frac{1}{2} \epsilon^{2}(n-2)(n-1) p^{n-3} x^{2}+\right. \\
\frac{1}{6} \epsilon^{3}(n-3)(n-2)(n-1) p^{n-4} x^{3}-p^{n-1}-\epsilon(n-1) p^{n-2} y- \\
\left.-\frac{1}{2} \epsilon^{2}(n-2)(n-1) p^{n-3} y^{2}-\frac{1}{6} \epsilon^{3}(n-3)(n-2)(n-1) p^{n-4} y^{3}\right)= \\
=\frac{1}{2}\left(\epsilon^{2} p^{n-3}\left(y^{2}-x^{2}\right)+\epsilon^{3} \frac{2(n-3)}{3} p^{n-4}\left(y^{3}-x^{3}\right)\right)
\end{gathered}
$$

Now, substitute the final expression of the equation (E.8) into the right-hand side of the equation (E.2), dividing both parts by $\frac{1}{2}(\epsilon y)^{2}(p+\epsilon y)^{n-3}$ and expanding $(p+\epsilon y)^{n-3}$ in the denominator of the right-hand side:

$$
y^{\prime}+\epsilon y^{\prime}=\frac{\epsilon^{2} p^{n-3}\left(y^{2}-x^{2}\right)+\epsilon^{3} \frac{2(n-3)}{3} p^{n-4}\left(y^{3}-x^{3}\right)}{\left(p^{n-3}+\epsilon(n-3) p^{n-4} y\right)(\epsilon y)^{2}}
$$

It is readily seen that $\epsilon^{2}$ cancel out. Since $n$ is a fixed number of players, around the singularity point it is true that $n \gg y$. Therefore, I can apply geometric series transformation $\frac{1}{1-q}=1+q+q^{2}+q^{3} \ldots$ to the polynomial in the denominator (note that the order of $\epsilon$ has to be consistent, and since the latter is multiplied by $\epsilon^{2}$, the expanded order coincides with the one truncated in the numerator):

$$
\frac{1}{p^{n-3}} \frac{1}{\left(1-\epsilon \frac{3-n}{p} y\right)}=\frac{1}{p^{n-3}}\left(1-\frac{\epsilon(n-3) y}{p}\right)
$$


Note, that I truncate the series after the second term, because the third term already includes $\epsilon^{2}$, which is higher than the order of $\epsilon$ received for equation (E.9). Therefore, equation (E.9) transforms into:

$$
y^{\prime}+\epsilon y^{\prime}=\frac{p^{n-3}\left(y^{2}-x^{2}\right)+\epsilon \frac{2(n-3)}{3} p^{n-4}\left(y^{3}-x^{3}\right)}{p^{n-3} y^{2}}\left(1-\frac{\epsilon(n-3) y}{p}\right)
$$

Since I have already canceled out $\epsilon^{2}$ in both parts of the equation, the newly truncated order is just $\epsilon$, so I drop any term with the power of $\epsilon$ greater than 1 . Hence, the right-hand side of the equation (E.11) becomes:

$$
\begin{gathered}
\frac{y^{2}-x^{2}}{y^{2}}+\epsilon \frac{2(n-3)\left(y^{3}-x^{3}\right)}{3 p y^{2}}\left(1-\frac{\epsilon(n-3) y}{p}\right)= \\
\frac{y^{2}-x^{2}}{y^{2}}+\epsilon \frac{2(n-3)\left(y^{3}-x^{3}\right)}{3 p y^{2}}-\epsilon \frac{y^{2}-x^{2}}{y^{2}} \frac{(n-3) y}{p}-\epsilon^{2} \frac{2(n-3)^{2} y\left(y^{3}-x^{3}\right)}{3 p^{2} y}= \\
\frac{y^{2}-x^{2}}{y^{2}}+\epsilon \frac{(3-n)(y-x)^{2}(2 x+y)}{3 p y^{2}}
\end{gathered}
$$

Note, that the term with $\epsilon^{2}$ has been dropped and each order of the variable(s) corresponds to the order of $\epsilon$. Thus, I have transformed equation (E.2) into an equation consisting of a leading order and one perturbed term ${ }^{3}$ :

$$
y_{0}^{\prime}+\epsilon y_{1}^{\prime}=\frac{y^{2}-x^{2}}{y^{2}}+\epsilon \frac{(3-n)(y-x)^{2}(2 x+y)}{3 p y^{2}}
$$

The leading order is the term with $\epsilon^{0}$, i.e. (capital letters here are used solely

\footnotetext{
${ }^{3}$ To receive additional perturbed terms add more orders of binomial expansion in the beginning.
} 
to emphasize the leading nature of the equation):

$$
\frac{d Y}{d X}=\frac{Y^{2}-X^{2}}{Y^{2}}
$$

The equation above is exactly the same as I solved for the case of 3 bidders.

Knowing the solution to the leading order, I can solve for a correction or perturbed term. To do this, terms on both sides have to be balanced:

$$
\epsilon^{0} y_{0}^{\prime}+\epsilon^{1} y_{1}^{\prime}+\ldots+\epsilon^{n} y_{n}^{\prime}=\epsilon^{0}(.)+\epsilon^{1}(.)+\ldots \epsilon^{n}(.)
$$

I have already found the solution to the leading term ODE $y_{0}^{\prime}=($.$) . Now,$ plug the symbolic conceived solution including the perturbed term into the equation (E.13):

$$
y_{0}^{\prime}+\epsilon y_{1}^{\prime}=\frac{\left(y_{0}+\epsilon y_{1}\right)^{2}-x^{2}}{\left(y_{0}+\epsilon y_{1}\right)^{2}}+\epsilon \frac{(3-n)\left(2 x+y_{0}+\epsilon y_{1}\right)\left(y_{0}+\epsilon y_{1}-x\right)^{2}}{3 p\left(y_{0}+\epsilon y_{1}\right)^{2}}
$$

Next, move the denominator to the numerator by the same way as before ${ }^{4}$. Within the set limit for $\epsilon$ I am only interested in the first two elements of the square of the sum of the first two terms: $(1+2 q)$, because all other terms will have higher orders. Hence, the denominator of the right-hand side can be rewritten as:

$$
\frac{1}{\left(y_{0}+\epsilon y_{1}\right)^{2}}=\frac{1}{y_{0}^{2}}\left(1-\frac{2 \epsilon y_{1}}{y_{0}}\right)
$$

\footnotetext{
${ }^{4}$ Note, that here it is squared, therefore $\left(\frac{1}{1-q}\right)^{2}=\left(1+q+q^{2}+q^{3} \ldots\right)^{2}$.
} 
Multiplying the transformed denominator on the equation (E.16) and dropping terms with $\epsilon$ higher than the order one I get:

$$
\begin{gathered}
\left(1-\frac{2 \epsilon y_{1}}{y_{0}}\right)\left(\frac{\left(-x^{2}+\left(y_{0}+\epsilon y_{1}\right)^{2}\right)}{y_{0}^{2}}-\frac{\epsilon(n-3)\left(-x+y_{0}+\epsilon y_{1}\right)^{2}\left(2 x+y_{0}+\epsilon y_{1}\right)}{3 y_{0}^{2} p}\right)= \\
=\frac{y_{0}^{2}-x^{2}}{y_{0}^{2}}+\epsilon \frac{1}{3 y_{0}^{2}}\left(\frac{(3-n) y_{0}^{3}+3(n-3) x^{2} y_{0}-2(n-3) x^{3}+}{p}+\frac{6 x^{2} y_{1}}{y_{0}}\right)+ \\
\quad+\epsilon^{2} \frac{1}{3 y_{0}^{2}}\left(\frac{4(n-3) x^{3} y_{1}}{p y_{0}}+\frac{(3-n) y_{0}^{2} y_{1}}{p}-\frac{3(n-3) x^{2} y_{1}}{p}-9 y_{1}^{2}\right)+ \\
+\epsilon^{3} \frac{1}{3 y_{0}^{2}}\left(\frac{3(n-3) y_{0} y_{1}^{2}}{p}-\frac{6(n-3) x^{2} y_{1}^{2}}{p y_{0}}-\frac{6 y_{1}^{3}}{y_{0}}\right)+\epsilon^{4} \frac{5(n-3) y_{1}^{3}}{3 p y_{0}^{2}}+\epsilon^{5} \frac{2(n-3) y_{1}^{4}}{3 p y_{0}^{3}}= \\
=\frac{y_{0}^{2}-x^{2}}{y_{0}^{2}}+\epsilon \frac{6 x^{3} y_{0}-2 n x^{3} y_{0}-9 x^{2} y_{0}^{2}+3 n x^{2} y_{0}^{2}+3 y_{0}^{4}-n y_{0}^{4}+6 p x^{2} y_{1}}{3 p y_{0}^{3}}
\end{gathered}
$$

Thus, the ODE for the perturbed term is (note, that it is linear in $y_{1}$ ):

$$
y_{1}^{\prime}=\frac{6 x^{3} y_{0}-2 n x^{3} y_{0}-9 x^{2} y_{0}^{2}+3 n x^{2} y_{0}^{2}+3 y_{0}^{4}-n y_{0}^{4}+6 p x^{2} y_{1}}{3 p y_{0}^{3}}
$$

Substituting $y_{0}=-0.754878 x$ into the equation above I get:

$$
y_{1}^{\prime}=-\frac{0.775\left(-8.684 x^{4}+2.895 n x^{4}+6 p x^{2} y_{1}\right)}{p x^{3}}
$$

This linear ODE can be easily solved for:

$$
y_{1}=\frac{(1.012-0.3373 n) x^{2}}{p}
$$

Adding both terms together I receive the final approximate solution for the 
equation (E.2):

$$
y=-0.755 x+\frac{(1.012-0.3373 n) x^{2}}{p}
$$

Note, that this equation is adjusted to the center of coordinates, it has negative slope $(n \geq 3)$ and each power of a variable maps into the corresponding order of the true polynomial.

Substituting back $Y=z(v)-p$ and $X=v-p$ and setting $r=1.012-0.3373 n$ to simplify the expression produces the final equation:

$$
z(v)=\left(\frac{7}{4}+r\right) p-\left(\frac{3}{4}+2 r\right) v+\frac{r v^{2}}{p}
$$

